\title{
IRREDUCIBLE BIMODULES OVER ALTERNATIVE ALGEBRAS AND SUPERALGEBRAS
}

\author{
IVAN SHESTAKOV AND MARIA TRUSHINA
}

Abstract. The aim of the paper is to describe irreducible birepresentations of alternative algebras and superalgebras. The complete classification is obtained for irreducible even bimodules of arbitrary dimension and characteristic and for finite-dimensional irreducible superbimodules over an algebraically closed field. We also describe irreducible superbimodules of any dimension and characteristic over the simple alternative superalgebras.

\section{An introduction}

Alternative algebras are the nearest generalization of associative algebras. An algebra $A$ is called alternative if it satisfies the identities

$$
\begin{aligned}
& (x, x, y)=0 \text { (left alternativity) } \\
& (x, y, y)=0 \text { (right alternativity) }
\end{aligned}
$$

where $(x, y, z)=(x y) z-x(y z)$ is the associator of the elements $x, y, z$. A classical example of non-associative alternative algebra is the 8-dimensional algebra of octonions (Cayley numbers), or, more generally, a Cayley-Dickson algebra.

The structure theory of finite-dimensional alternative algebras was developed by M. Zorn: if $A$ is a finite-dimensional alternative algebra, then $A$ contains a unique maximal nilpotent ideal $R$ (the nil radical of the algebra $A$ ), and the quotient algebra $A / R$ is semisimple 32 . Every semisimple algebra is a direct sum of ideals which are simple algebras 31. Every simple alternative algebra is either an associative algebra or a Cayley-Dickson algebra over its center (see [15, 31]). For principal results of the theory of infinite-dimensional alternative algebras we refer the reader to 30 .

The theory of representations of alternative algebras was initiated in the papers by R. D. Schafer [16] and N. Jacobson [8], where birepresentations of finitedimensional alternative algebras were studied. In particular, in [16] it has been shown that any birepresentation of a semisimple alternative algebra of characteristic 0 (without any restriction on dimension) is completely reducible, and in 8 the irreducible alternative bimodules over finite-dimensional algebras were classified. It should be noted that, contrary to the associative case, for non-associative algebras the concept of birepresentation is more natural and easily defined than that of onesided (right or left) representation. Right representations of alternative algebras

Received by the editors January 12, 2014 and, in revised form, May 13, 2014.

2010 Mathematics Subject Classification. Primary 17A60, 17A70, 17D05; Secondary 16D90, $16 \mathrm{D} 60$.

The first author was supported by FAPESP, Proc. 2010/50347-9 and CNPq, Proc. 3305344/ 2009-9.

The second author was supported by FAPESP, Proc. 2008/50141-1. 
were studied by K. A. Ževlakov [29] and A. M. Slin'ko and I. P. Shestakov [22]. In particular, in 22] some description of right irreducible alternative modules was obtained and a characterization of the quasi-regular radical in terms of representation theory was given. However, even in the finite-dimensional case the classification of irreducible alternative right modules remains incomplete. For example, until now it is not clear whether the left regular representation of a Cayley-Dickson algebra (it is irreducible) is an alternative right representation.

The simple alternative superalgebras were described by E. I. Zel'manov and I. P. Shestakov [28] (in the case of characteristic different from 2 and 3) and I. P. Shestakov [19] (for arbitrary characteristic). It turned out that non-trivial examples of such superalgebras appear in the case of characteristic 2 or 3 only. The study of representations of alternative superalgebras began in the work of N. A. Pisarenko [11]. In that paper the finite-dimensional irreducible alternative superbimodules over finite-dimensional simple alternative superalgebras of characteristic different from 2 and 3 were described. It turned out that in this case non-trivial alternative non-associative superbimodules appear in the case of the minimal non-trivial simple superalgebra, i.e., doubled field $F[u]=F \oplus F u, u^{2}=1$ only. In 9,24 irreducible bimodules over the simple alternative superalgebras $B(1 \mid 2)$ and $B(4 \mid 2)$ of characteristic 3 and of dimensions 3 and 6 were described.

The aim of our paper is to describe irreducible birepresentations of alternative algebras of arbitrary dimension and characteristic on one side, and, on the other, to describe finite-dimensional irreducible alternative superbimodules. We also describe irreducible superbimodules of any dimension and characteristic over the simple alternative superalgebras. To complete the description of irreducible infinite-dimensional superbimodules, it remains to describe such superbimodules (or to prove their absence) over prime locally nilpotent alternative superalgebras of characteristic 3 .

The results of the paper were announced in [25].

\section{Definitions AND KNOWn RESUlts}

2.1. Birepresentations of alternative algebras. Let $A$ be an alternative algebra over a field $F$ and let $V$ be an $A$-bimodule, i.e., $V$ is a vector space over $F$ such that on $V$ left and right bilinear multiplications by the elements of the algebra $A$ are defined:

$$
A \otimes_{F} V \rightarrow V, \quad(a \otimes v) \mapsto a \cdot v, \quad V \otimes_{F} A \rightarrow V, \quad(v \otimes a) \mapsto v \cdot a,
$$

where $a \in A, v \in V$. In the vector space direct sum $E=A \oplus V$ define a product $*$ by

$$
\left(a_{1}+v_{1}\right) *\left(a_{2}+v_{2}\right)=a_{1} a_{2}+a_{1} \cdot v_{2}+v_{1} \cdot a_{2},
$$

where $a_{1}, a_{2} \in A, v_{1}, v_{2} \in V$. So $E$ becomes an algebra over $F$, in which $A$ is a subalgebra and $V$ is an ideal with zero multiplication. The algebra $E$ is called the split null extension of the algebra $A$ by its bimodule $V$. The bimodule $V$ is called an alternative $A$-bimodule, if the split null extension $E=A \oplus V$ is an alternative algebra. It is easy to check that a bimodule $V$ is alternative if and only if for any $a, b \in A, v \in V$ the following equalities hold in the algebra $E$ :

$$
(a, b, v)=(b, v, a)=(v, a, b), \quad(v, a, a)=0 .
$$

The description of irreducible (bi)modules is one of the main problems in the (bi)representation theory in any class of algebras. For an algebra $A$ denote by 
$\operatorname{Reg} A$ the regular bimodule $V:=A$ with the action of $A$ given by the product in $A$. It is clear that the bimodule Reg $\mathbb{O}$ over a Cayley-Dickson algebra $\mathbb{O}$ is an irreducible faithful alternative bimodule which is not associative. Further examples of alternative non-associative bimodules are provided by the so-called Cayley bimodules over a generalized quaternion algebra $\mathbb{H}$, which are defined as follows. Let $a \mapsto \bar{a}$ be the symplectic involution in $\mathbb{H}$; then an alternative $\mathbb{H}$-bimodule $V$ is called a Cayley bimodule if it satisfies the identity $a v=v \bar{a}$ for any $a \in \mathbb{H}, v \in V$. The Cayley H-bimodules form a category which is isomorphic to the category of left associative $\mathbb{H}$-modules via the following isomorphism (see [19, Lemmas 11,12]). Let $L$ be a left associative $\mathbb{H}$-module with the action $(a, v) \mapsto a v$. Then the vector space $L$ with the bimodule operations

$$
v \cdot a=a v, \quad a \cdot v=\bar{a} v,
$$

is an alternative Cayley bimodule over $\mathbb{H}$ which we will denote as Cay $L$.

The results of the papers of R. D. Schafer [16] and N. Jacobson [8] mentioned in the introduction lead to the following description of finite-dimensional irreducible alternative bimodules.

Theorem 2.1. Let $A$ be a finite-dimensional alternative algebra over a field $F$ of characteristic different from 2 and let $V$ be an irreducible alternative faithful A-bimodule. Then one of the following cases holds:

- $A$ is associative and $V$ is an associative bimodule,

- $A=\mathbb{O}$ and $V=\operatorname{Reg} \mathbb{O}$,

- $A=\mathbb{H}$ and $V=$ Cay $L$, where $L$ is a minimal left ideal of $\mathbb{H}$.

Irreducible bimodules over generalized quaternion algebras were described by N. Jacobson 8 under the assumption that the ground field has characteristic different from 2 and centralizes the bimodule. In [19] the first author extended this result, removing the restrictions on the characteristic, dimension, and the condition of centralization.

2.2. Alternative superalgebras. An algebra $A$ is called a $\mathbb{Z}_{2}$-graded algebra or superalgebra, if $A=A_{\overline{0}} \oplus A_{\overline{1}}$, where $A_{i} A_{j} \subseteq A_{i+j}, i, j \in \mathbb{Z}_{2}$. The subspaces $A_{\overline{0}}$ and $A_{\overline{1}}$ are called respectively the even and odd parts of the superalgebra $A$. For example, the Grassmann algebra $G=G_{\overline{0}} \oplus G_{\overline{1}}$ becomes a superalgebra, if we denote by $G_{\overline{0}}\left(G_{\overline{1}}\right)$ the submodule generated by the words of even (odd) length from generators of the algebra $G$. A superalgebra $A=A_{\overline{0}} \oplus A_{\overline{1}}$ is called an alternative superalgebra, if its Grassmann envelope $G(A):=G_{\overline{0}} \otimes A_{\overline{0}} \oplus G_{\overline{1}} \otimes A_{\overline{1}}$ is an alternative algebra. From this definition it follows that the alternative superalgebras are defined by the identities

$$
\begin{aligned}
& \left(a_{i}, a_{j}, a_{k}\right)=(-1)^{j k+1}\left(a_{i}, a_{k}, a_{j}\right), \\
& \left(a_{i}, a_{j}, a_{k}\right)=(-1)^{i j+1}\left(a_{j}, a_{i}, a_{k}\right), \\
& \left(a_{0}, a_{0}, A\right)=0,
\end{aligned}
$$

where $a_{s} \in A_{s}, s=i, j, k \in\{\overline{0}, \overline{1}\}$.

Observe that an alternative superalgebra is not the same as an alternative $\mathbb{Z}_{2^{-}}$ graded algebra: the simple alternative superalgebras of characteristic 3 below are not alternative algebras. 
Let $A$ be an algebra. Denote by $A[u]$ the superalgebra obtained by doubling of the algebra $A$ :

$$
A[u]:=A \oplus A \cdot u, \quad(A[u])_{\overline{0}}=A, \quad(A[u])_{\overline{1}}=A \cdot u,
$$

where $u$ is an odd central element, $u^{2}=\alpha \in F, \alpha \neq 0$. When $u^{2}=1$, we will denote $A[u]$ as $A[\sqrt{1}]$.

C. T. C. Wall [26] proved that every simple finite-dimensional associative superalgebra over an algebraically closed field $F$ is isomorphic to one of the following superalgebras:

$$
\text { - } A=\mathrm{M}_{m \mid n}(F), \quad A_{\overline{0}}=\left\{\left(\begin{array}{cc}
\star & 0 \\
0 & \star
\end{array}\right) \begin{array}{c}
m \\
n
\end{array}\right\}, \quad A_{\overline{1}}=\left\{\left(\begin{array}{cc}
0 & \star \\
\star & 0
\end{array}\right) \begin{array}{c}
m \\
n
\end{array}\right\},
$$

- $A=\mathrm{M}_{n}(F)[\sqrt{1}]$, the doubled matrix algebra.

In 28, it was proved that every non-trivial simple alternative superalgebra of characteristic different from 2 and 3 is associative. In particular, the non-trivial simple finite-dimensional alternative superalgebras over an algebraically closed field $F$ of characteristic different from 2,3 are exhausted by the superalgebras $\mathrm{M}_{m \mid n}$, $\mathrm{M}_{n}[\sqrt{1}]$.

The simple alternative superalgebras of characteristic 2 and 3 were described in [19]. In these cases non-associative non-trivial simple superalgebras appeared.

Over a field of characteristic 2, any such superalgebra is isomorphic to one of the following two superalgebras obtained from a Cayley-Dickson algebra:

- the Cayley-Dickson superalgebra $\mathbb{O}(4 \mid 4)=\mathbb{O}:=\mathbb{H} \oplus v \mathbb{H}$ with the $\mathbb{Z}_{2^{-}}$ grading induced by applying the Cayley-Dickson process to the generalized quaternion subalgebra $\mathbb{H}$,

- the doubled Cayley-Dickson algebra $\mathbb{O}[u]$.

Note that both these superalgebras are alternative algebras.

A simple non-associative non-trivial alternative superalgebra of characteristic 3 is isomorphic to one of the following superalgebras (see [18, 19]):

- superalgebra $B(1 \mid 2)$. Let $V$ be a 2-dimensional vector space over a field $F$ with a non-zero skew-symmetric bilinear form $\langle\cdot, \cdot\rangle$. The unital superalgebra $B(1 \mid 2):=F \cdot 1 \oplus V$ with an identity 1 is given by the grading

$$
B(1 \mid 2)_{\overline{0}}=F \cdot 1, \quad B(1 \mid 2)_{\overline{1}}=V
$$

and the supercommutative multiplication $x y=\langle x, y\rangle \cdot 1$ for $x, y \in V$;

- superalgebra $B(4 \mid 2)$. Let $V$ be the same space as above; set

$$
B(4 \mid 2)_{\overline{0}}:=\text { End } V \cong \mathrm{M}_{2}(F), \quad B(4 \mid 2)_{\overline{1}}:=V
$$

with the multiplication

$$
v \cdot a=a(v)=\bar{a} \cdot v, \quad u \cdot v=\langle\cdot, u\rangle v \in \operatorname{End} V,
$$

where $a \in$ End $V, u, v \in V$ and $a \mapsto \bar{a}$ denote the symplectic involution in End $V$ (i.e. $\langle a(u), v\rangle=\langle u, \bar{a}(v)\rangle$ for any $u, v \in V$ ). It is easy to check that $V=B(4 \mid 2)_{\overline{1}}$ is a Cayley bimodule over $\mathrm{M}_{2}(F)=B(4 \mid 2)_{\overline{0}}$; 
- twisted superalgebra of vector type $B(\Gamma, D, \gamma)$. Let $\Gamma$ be an associative commutative algebra over a field $F$, let $D$ be a non-zero derivation of $\Gamma$, $\gamma \in \Gamma$. Denote by $\bar{\Gamma}$ an isomorphic copy of the vector space $\Gamma$ with respect to the isomorphism $a \mapsto \bar{a}$ and set $B(\Gamma, D, \gamma):=\Gamma \oplus \bar{\Gamma}$ with the multiplication

$$
\begin{aligned}
& a \cdot b=a b, \\
& a \cdot \bar{b}=\bar{a} \cdot b=\overline{a b}, \\
& \bar{a} \cdot \bar{b}=\gamma a b+2 D(a) b+a D(b),
\end{aligned}
$$

where $a, b \in \Gamma, a b$ is the product of the elements $a$ and $b$ in $\Gamma$, and with the grading

$$
B(\Gamma, D, \gamma)_{\overline{0}}=\Gamma, \quad B(\Gamma, D, \gamma)_{\overline{1}}=\bar{\Gamma}
$$

The superalgebra $B(\Gamma, D, \gamma)$ is simple if and only if the algebra $\Gamma$ does not contain proper $D$-invariant ideals (i.e. $D$-simple).

2.3. Alternative superbimodules. The alternative superbimodules are defined similarly to the non-graded case. A bimodule $V=V_{\overline{0}} \oplus V_{\overline{1}}$ over an alternative superalgebra $A=A_{\overline{0}} \oplus A_{\overline{1}}$ is called an alternative $A$-superbimodule, if the split null extension $A \oplus V=\left(A_{\overline{0}} \oplus V_{\overline{0}}\right) \oplus\left(A_{\overline{1}} \oplus V_{\overline{1}}\right)$ is an alternative superalgebra.

We will call an $A$-superbimodule $V^{\mathrm{op}}=V_{\overline{0}}^{\mathrm{op}}+V_{\overline{1}}^{\mathrm{op}}$ opposite to an $A$-superbimodule $V=V_{\overline{0}}+V_{\overline{1}}$, if $V_{\overline{0}}^{\mathrm{op}}=V_{\overline{1}}, V_{\overline{1}}^{\mathrm{op}}=V_{\overline{0}}$ and $A$ acts on it by the following rule: $a \cdot v=(-1)^{|a|} a v, v \cdot a=v a$, where $v \in V^{\mathrm{op}}, a \in A_{\overline{0}} \cup A_{\overline{1}}$, and $|a|$ means the parity of $a$, that is, $|a|=i$ if $a \in A_{i}, i=\overline{0}, \overline{1}$.

It is easy to check that for any superbimodule $V$ the identity map $V \rightarrow V^{\mathrm{op}}, v \mapsto$ $v$, is an odd isomorphism between $V$ and $V^{\mathrm{op}}$. In particular, if $V$ is alternative, the opposite superbimodule $V^{\text {op }}$ is alternative as well. We sometimes will say that the bimodule $V^{\text {op }}$ is obtained from $V$ by changing of parity.

As in the case of algebras, the main problem of the theory of representations of superalgebras is the description of irreducible superbimodules. For alternative algebras, such bimodules are always defined either over simple algebras or over a direct sum of two simple algebras, and in the latter case the bimodule is associative. It is an important difference of the supercase that there are irreducible superbimodules over nilpotent superalgebras. This first was noted in [18, where this fact was used to construct finite-dimensional solvable non-nilpotent alternative and Jordan superalgebras.

Let $A=A_{\overline{1}}=F x, x^{2}=0$. Assume that the field $F$ contains a primitive cube root of 1, i.e., such element $\varepsilon$, that $\varepsilon^{2}+\varepsilon+1=0$. Consider the $A$-bimodule $V^{\varepsilon}(1 \mid 1)=F v_{0} \oplus F v_{1}$, where $V_{\overline{0}}=F v_{0}, V_{\overline{1}}=F v_{1}$ and the action of the element $u$ is given by the equalities

$$
v_{0} \cdot x=v_{1}, \quad x \cdot v_{0}=\varepsilon v_{1}, \quad v_{1} \cdot x=v_{0}, \quad x \cdot v_{1}=(\varepsilon+1) v_{0} .
$$

It is easy to check that $V^{\varepsilon}(1 \mid 1)$ is an irreducible alternative superbimodule; furthermore, $V^{\varepsilon}(1 \mid 1)^{\text {op }} \cong V^{\varepsilon^{2}}(1 \mid 1)$.

Note that the commutator bimodule $V^{\varepsilon}(1 \mid 1)^{(-)}$over the Malcev superalgebra $A^{(-)}$appeared in the paper by A. Elduque and the first author [7]. 
It is clear that the bimodule $V^{\varepsilon}(1 \mid 1)$ can be considered also as a unital superbimodule over the superalgebra $A^{\sharp}=F \oplus F x$ obtained from $A$ by adjoining an identity 1; obviously, it remains irreducible.

If the field $F$ does not contain a primitive cube root of 1 and $\operatorname{char} F \neq 3$, then a 4-dimensional irreducible alternative superbimodule over $A$ can be constructed (see [18]).

It is clear that for any simple alternative superalgebra $A$ the regular superbimodule $\operatorname{Reg} A$ and its opposite companion $(\operatorname{Reg} A)^{\text {op }}$ are irreducible alternative superbimodules.

N. A. Pisarenko [1] investigated the structure of alternative superbimodules over finite-dimensional semisimple superalgebras of characteristic different from 2,3 .

We call $V$ non-trivial, if $V_{\overline{0}} \neq 0$ and $V_{\overline{1}} \neq 0$.

Theorem 2.2 ([1]). Let $A$ be a finite-dimensional semisimple alternative superalgebra over an algebraically closed field $F$ of characteristic different from 2, 3. Thus $A=A_{1} \oplus A_{2} \oplus \ldots \oplus A_{k}$, where each summand $A_{i}$ is isomorphic to either a Cayley-Dickson algebra $\left(0\right.$ or a matrix superalgebra of types $\mathrm{M}_{m \mid n}$ and $\mathrm{M}_{k}[\sqrt{1}]$ over F. Then

- any alternative superbimodule over $A$ is a direct sum of associative $\left(A_{i}, A_{j}\right)$ bimodules and unital alternative $A_{i}$-bimodules;

- any non-trivial faithful irreducible alternative superbimodule $V$ over the simple superalgebra $A_{i}$ is either associative and isomorphic to one of the bimodules $\operatorname{Reg} \mathrm{M}_{m \mid n},\left(\operatorname{Reg} \mathrm{M}_{m \mid n}\right)^{\mathrm{op}}, \operatorname{Reg}\left(\mathrm{M}_{k}[\sqrt{1}]\right), \operatorname{Reg}\left(\mathrm{M}_{k}[\sqrt{1}]\right)^{\mathrm{op}}$, or $A_{i}=F[\sqrt{1}]=F[u], \operatorname{dim} V=2, V_{\overline{0}}=F v_{0}, V_{\overline{1}}=F v_{1}$ and the (unital) action of $A_{i}$ on $V$ has one of the following forms:

(1) $v_{0} u=v_{1}, v_{1} u=0, u v_{0}=-2 v_{1}, u v_{1}=-v_{0}$,

(2) $v_{0} u=v_{1}, v_{1} u=2 v_{0}, u v_{0}=0, u v_{1}=v_{0}$,

(3) $v_{0} u=\frac{1+\alpha}{\delta} v_{1}, v_{1} u=\delta v_{0}, u v_{0}=v_{1}, u v_{1}=(1-\alpha) v_{0}$,

where $\alpha, \delta \in F$ are non-zero roots of the equation $\alpha^{2}+\alpha \delta+\delta^{2}=1$.

Remark 2.1. Note that in the theorem above, the bimodules of types (1) - (3) can be defined in a unified manner as the following series of unital bimodules over $F[\sqrt{1}]=F \oplus F u$ :

$$
V=V_{\lambda, \mu}(1 \mid 1)=F v_{0} \oplus F v_{1}, \quad V_{\overline{0}}=F v_{0}, \quad V_{\overline{1}}=F v_{1},
$$

with the action

$$
v_{0} u=(3 \mu-\lambda) v_{1}, \quad u v_{0}=2 \lambda v_{1}, \quad v_{1} u=2 \mu v_{0}, \quad u v_{1}=(\lambda+\mu) v_{0},
$$

where $\lambda, \mu \in F, \lambda^{2}+3 \mu^{2}=1$. In fact, the bimodule of type (1) is the bimodule $V_{-1,0}(1 \mid 1)$, the bimodule of type (2) is isomorphic to the bimodule $V_{0,1 / \sqrt{3}}(1 \mid 1)$, the bimodules of type (3) are isomorphic to $V_{\lambda, \mu}(1 \mid 1)$ for $\lambda=\sqrt{2-2 \alpha-\delta} / 2$, $\mu=\delta /(4 \lambda)$.

The irreducible unital superbimodules over the superalgebras $B(1 \mid 2)$ and $B(4 \mid 2)$ were described by M. C. López-Díaz and the first author in 9 . (The classification of irreducible finite-dimensional alternative superbimodules over $B(1 \mid 2)$ was also obtained independently by the second author in [24.) 
Theorem 2.3 (9, 24]). Any irreducible alternative bimodule over the superalgebra $B=B(1 \mid 2)$, which is not associative, is isomorphic either to $\operatorname{Reg} B$, or to $(\operatorname{Reg} B)^{\mathrm{op}}$, or belongs to the series of irreducible bimodules

$$
\begin{gathered}
V(\lambda, \mu)(3 \mid 3)=V_{\overline{0}} \oplus V_{\overline{1}}, \\
V_{\overline{0}}=\operatorname{Vect}_{F}\left\langle v_{0}, v_{1} R_{y}, v_{0} R_{y}^{2}\right\rangle, \quad V_{\overline{1}}=\operatorname{Vect}_{F}\left\langle v_{1}, v_{0} R_{y}, v_{1} R_{y}^{2}\right\rangle,
\end{gathered}
$$

with the action

$$
\begin{aligned}
& v R_{y}^{j} \cdot y= \begin{cases}v R_{y}^{j+1}, & j<2, \\
\mu v^{s}, & j=2,\end{cases} \\
& v R_{y}^{j} \cdot x=\lambda v^{s} R_{y}^{j}+j v R_{y}^{j-1}, \quad j=0,1,2,
\end{aligned}
$$

where $v \in\left\{v_{0}, v_{1}\right\}, v_{i}^{s}=v_{1-i}$ and $\lambda, \mu$ are non-zero scalars. In addition, the superbimodules $V(\lambda, \mu)$ and $V\left(\lambda^{\prime}, \mu^{\prime}\right)$ are isomorphic if and only if $(\lambda, \mu)= \pm\left(\lambda^{\prime}, \mu^{\prime}\right)$. Moreover, $V(\lambda, \mu) \cong(V(\lambda, \mu))^{\text {op }}$.

Theorem 2.4 ([9]). Every alternative bimodule over the superalgebra $B(4 \mid 2)$ is completely reducible, and every irreducible bimodule up to the change of parity is isomorphic to the regular bimodule.

2.4. Useful identities. We recall some useful identities that hold in any alternative algebra (see [5], [19], 30]):

$$
\begin{aligned}
{[a b, c]=} & a[b, c]+[a, c] b+3(a, b, c), \\
(a b, c, d)= & a(b, c, d)+(a, c, d) b-(a, b,[c, d]), \\
{[(a, b, c), d]=} & (a b, c, d)+(b c, a, d)+(c a, b, d), \\
((c, a, b), a, b)= & {[a, b](c, a, b), } \\
((a, b, c), x, y)= & ((a, x, y), b, c)+(a,(b, x, y), c)+(a, b,(c, x, y)) \\
& -[b,(a, c,[x, y])]+([a, c], b,[x, y]), \\
(z, x, t y)= & -(z, t, x y)+(z, x, y) t+(z, t, y) x, \\
(z, x, y t)= & -(z, t, y x)+x(z, t, y)+t(z, x, y) .
\end{aligned}
$$

We will also need the identities

$$
\begin{aligned}
z(y x y) & =((z y) x) y, \\
(z y)(x z) & =(z(y x)) z,
\end{aligned}
$$

known as right and central Moufang identities (see [30]).

We will often indicate the use of these equations by an equation number above an equals sign.

\section{Reduction to prime superalgebras and superalgebras With $A_{\overline{1}}^{2}=0$}

Let $A$ be an alternative superalgebra and let $V$ be an irreducible $A$-superbimodule. Denote by Ann $V$ the annihilator of $V$ :

$$
\text { Ann } V:=\{a \in A \mid a \cdot V=V \cdot a=0\} .
$$

It is easy to see that Ann $V$ is an ideal of $A$ and $V$ is an irreducible faithful bimodule over $A$ /Ann $V$. A bimodule $V$ is called associative if $(V, A, A)=0$. One can easily check that if $V$ is associative, then $(A, A, A) \subseteq$ Ann $V$, hence faithful associative bimodules exist only over associative algebras. 
Below $V$ always will denote an irreducible faithful $A$-superbimodule which is not associative. We will use the following notation:

- $E=E(A, V)=A \oplus V$, the split null extension;

- $(\lambda, \rho)$, the birepresentation of $A$ associated with $V$, that is, $\lambda, \rho$ are linear mappings from $A$ to End $V$ such that for any $a \in A, v \in V$ holds

$$
\lambda(a): v \mapsto(-1)^{|a||v|} a \cdot v, \quad \rho(a): v \mapsto v \cdot a ;
$$

- $\mathcal{Z}(V)=\mathcal{Z}_{A}(V):=\left\{\varphi \in\right.$ End $\left.V \mid[\varphi, \rho(a)]_{s}=[\varphi, \lambda(a)]_{s}=0 \forall a \in A\right\}$, the centralizer of the $A$-bimodule $V$, where $[a, b]_{s}=a b-(-1)^{|a||b|} b a$;

- $\mathcal{E}:=\mathcal{Z}(\operatorname{Reg} E)$, the supercentroid of $E$;

- $\Gamma:=\{\alpha \in \mathcal{E} \mid V \alpha \subseteq V, A \alpha \subseteq A\}$.

Proposition 3.1 ([7). The centralizer $\mathcal{Z}=\mathcal{Z}(V)$ is a graded division algebra and $\Gamma$ is a commutative superalgebra which can be embedded in $\mathcal{Z}$ via the map $\pi:\left.\alpha \mapsto \alpha\right|_{V}$.

Proof. By the graded version of the Schur Lemma, for an irreducible bimodule $V$ the centralizer $\mathcal{Z}(V)$ is a graded division algebra. Consider the homomorphism $\pi: \Gamma \rightarrow$ End $V,\left.\alpha \mapsto \alpha\right|_{V}$, and let $\alpha \in \operatorname{ker} \pi$. Then $V(A \alpha)=(V \alpha) A=0$, hence $A \alpha=0$ and $\alpha=0$. It is clear also that $\pi(\Gamma) \subseteq \mathcal{Z}$. Furthermore, let $\alpha, \beta \in \Gamma, v \in V, x \in A$; then we have

$$
\begin{aligned}
((v \alpha) \beta) x & =(-1)^{|\beta||x|}(v \alpha)(x \beta)=(-1)^{|\beta||x|+|\alpha|(|x|+|\beta|)}(v(x \beta)) \alpha \\
& =(-1)^{|\alpha||x|+|\alpha||\beta|}((v \beta) x) \alpha=(-1)^{|\alpha||\beta|}((v \beta) \alpha) x .
\end{aligned}
$$

Therefore, $\left(V[\alpha, \beta]_{s}\right) A=0$, which implies that $[\alpha, \beta]_{s}=0$ and $\Gamma$ is a supercommutative subsuperalgebra of $\mathcal{Z}$.

Theorem 3.1. Let $A=A_{\overline{0}} \oplus A_{\overline{1}}$ be an alternative superalgebra. If there exists an irreducible faithful $A$-superbimodule $V$ which is not associative, then $A$ is prime or $A_{\overline{1}} \neq 0, A_{\overline{1}}^{2}=0$.

We first prove two lemmas.

Lemma 3.1. Let non-zero elements $a, b \in A_{\overline{0}} \cup A_{\overline{1}}$ satisfy $[a, b]_{s}=(a, A, b)=0$. Then the subset $(V, a, b)$ is an $A$-subbimodule of $V$. Moreover, if $a b=b a=0$ and at least one of the elements $a, b$ is even, then $(V, a, b)=0$.

Proof. In fact, for any $v \in V_{\overline{0}} \cup V_{\overline{1}}, r \in A_{\overline{0}} \cup A_{\overline{1}}$ we have by (2) and its superization

$$
\begin{aligned}
& (v, a, b) r= \pm(v r, a, b) \pm v(r, a, b) \pm\left(v, r,[a, b]_{s}\right)= \pm(v r, a, b) \in(V, a, b), \\
& r(v, a, b)=(r v, a, b) \pm(r, a, b) v+\left(r, v,[a, b]_{s}\right)=(r v, a, b) \in(V, a, b),
\end{aligned}
$$

which proves that $(V, a, b)$ is a subbimodule of $V$. Now, let $a b=b a=0$ and $a \in A_{\overline{0}}$. If $(V, a, b) \neq 0$, then by irreducibility $(V, a, b)=V$. By the Moufang identities, we have

$$
\begin{aligned}
& a(v, a, b)=(v, a, b a)=0, \\
& (v, a, b) a=(v, a, a b)=0 .
\end{aligned}
$$

Therefore, $0 \neq a \in$ Ann $V$, a contradiction.

Lemma 3.2. Let non-zero ideals $I, J$ of $A$ satisfy $I J=J I=0$. Then $I+J \subseteq A_{\overline{1}}$. Moreover, if $A=A_{\overline{0}}$, then $A$ is prime. 
Proof. Assume that $I_{\overline{0}} \neq 0$. By the previous lemma, the set $N:=(V, I, J)$ is an $A$-subbimodule of $V$ and $\left(V, I_{\overline{0}}, J\right)=\left(V, I, J_{\overline{0}}\right)=0$. If $i \in I_{\overline{1}}, j \in J_{\overline{1}}, a \in I_{\overline{0}}$, then, by the Moufang identities, for any homogeneous $v \in V$,

$$
\begin{aligned}
(v, i, j) a & =(v, i, a j)+(v, a, i j)+(v, a, j) i=0, \\
a(v, i, j) & =(v, i, j a)-(v, a, j i)-(-1)^{|v|} i(v, a, j)=0 .
\end{aligned}
$$

Thus $0 \neq I_{\overline{0}} \subseteq \operatorname{Ann} N$, and $N=0$. Consider now the sets $I \cdot V, V \cdot I$. It is easy to see that they are subbimodules of $V$ and at least one of them is non-zero. Assume that $I \cdot V \neq 0$; then $I \cdot V=V$ and we have

$$
J \cdot V=J \cdot(I \cdot V) \subseteq(J I) \cdot V+(J, I, V)=0 .
$$

Similarly, if $V \cdot I \neq 0$, then we would have $V \cdot J=0$ and

$$
0 \neq J \subseteq \text { Ann } V=0,
$$

a contradiction. Therefore, $V \cdot I=0$, and consequently $(V, A, I)=0$. Furthermore, by the Moufang identity we have

$$
I \cdot(V, A, A) \subseteq(V, A, A I)+(V, I, A A)+A \cdot(V, I, A)=0,
$$

which proves that $(V, A, A) \subseteq \operatorname{Ann}_{V}(I):=\{v \in V \mid v \cdot I=I \cdot v=0\}$. It is clear that $\operatorname{Ann}_{V}(I)$ is a subbimodule of $V$ which should be zero since $I \neq 0$. Therefore, $(V, A, A)=0$ which contradicts the non-associativity of $V$, and this proves that $I_{\overline{0}}=0$. Similarly, $J_{\overline{0}}=0$. In particular, if $I$ is an ideal of $A$ with $I^{2}=0$, then $I \subseteq A_{\overline{1}}$.

We continue by considering the case $A=A_{\overline{0}}$. Assume that $I, J$ are ideals of $A$ with $I J=0$. Then $(I \cap J)^{2}=0, I \cap J \subseteq A_{\overline{1}}=0$, and we have $J I \subseteq I \cap J=0$. Therefore, $I=0$ or $J=0$, and $A$ is prime.

The proof of the theorem. Assume that $A_{\overline{1}}^{2} \neq 0$ and prove that in this case $A$ is prime. As above, it suffices to prove that if $I, J$ are ideals in $A$ with $I J=J I=0$, then $I=0$ or $J=0$. Assume that $I \neq 0, J \neq 0$; then by Lemma 3.2 , we have $I+J \subseteq$ $A_{\overline{1}}$. Therefore, $I A_{\overline{1}}+A_{\overline{1}} I \subseteq I_{\overline{0}}=0$ and $0 \neq A_{\overline{1}} \subseteq$ Ann $I$. Since the annihilator of an ideal in $A$ is an ideal, we may apply Lemma 3.2 to the ideals $I$, Ann $I$ and to get Ann $I \subseteq A_{\overline{1}}$. Then $A_{\overline{1}}^{2} \subseteq$ Ann $I \subseteq A_{\overline{1}}$ and $A_{\overline{1}}^{2}=0$, a contradiction.

\section{THE CASE $A_{\overline{1}}=0$}

The objective of this section is to generalize the results by N. Jacobson and R. D. Schafer on irreducible alternative bimodules given in Theorem 2.1 to arbitrary dimension and characteristic.

Theorem 4.1. Let $A$ be an alternative algebra and let $V$ be an irreducible alternative faithful A-bimodule which is not associative, both $A$ and $V$ to be of arbitrary dimension. Then one of the following cases holds:

- $A=\mathbb{O}$ and $V=\operatorname{Reg}(\mathbb{O}$,

- $A=\mathbb{H}$ and $V=$ Cay $L$, where $L$ is a minimal left ideal of $\mathbb{H}$.

Proof. By Theorem [3.1, $A$ is prime. Hence, by [30], $A$ is either an associative prime algebra or a Cayley-Dickson ring or is degenerate, that is, contains non-zero absolute zero divisors. (Recall that an element $a \in A$ is called an absolute zero divisor (a.z.d.) if $a A a=0$.) 
In the last case, let $0 \neq a \in A$ be an a.z.d. We next show that $a \in \operatorname{Ann} V$. Indeed, let $u=v \cdot a \neq 0$ for some $v \in V$. Then $V$ is generated by $u$. Let $(\lambda, \rho): A \rightarrow$ End $V$ be the birepresentation of $A$ associated with the $A$-bimodule $V$ and let $M(A)$ be the subalgebra of the associative algebra End $V$ generated by the set $\{\lambda(a), \rho(a) \mid a \in A\}$. Then $V=u M(A)$, and there exists $W \in M(A)$ such that $v=u W=v \rho(a) W=\cdots=v(\rho(a) W)^{n}$. From [17, Corollary of Lemma 1, Corollary 1 of Theorem 1], it follows that the operator $\rho(a) W$ is nilpotent. Hence $v=0$, a contradiction.

Therefore, $A$ is a prime associative algebra or a Cayley-Dickson ring. Let us prove that in the first case $A$ is a central order in a generalized quaternion algebra. Show first that $A$ is not commutative. In fact, if it were true, then by Lemma 3.1 for any $a, b \in A$ we would have an $A$-subbimodule $(V, a, b)$. Since $(V, A, A) \neq 0$, there exist $a, b \in A$ such that $V=(V, a, b)$. But then $V=(V, a, b)=((V, a, b), a, b)=$ $[a, b](V, a, b)=0$ by (4), a contradiction.

Let $V_{a s}:=\{v \in V \mid(v, A, A)=0\}$. Since $A$ is associative, it follows from (2) that $V_{a s}$ is a subbimodule of $V$ which should be zero since $V$ is irreducible and not associative. Since $A$ is prime and non-commutative, $A$ does not satisfy the identity $[x, y]^{4}=0$. In fact, otherwise $A$ would be a central order in a simple algebra which satisfies the same identity $[x, y]^{4}=0$ and hence is commutative, a contradiction (see, for instance, [14]). Let $a, b \in A$ such that $n=[a, b]^{4} \neq 0$. Assume that there exists $c \in A$ such that $[n, c] \neq 0$. By [30, Lemma 7.5], we have $((V[n, c], A, A), A, A)=0$. Therefore, $(V[n, c], A, A) \subseteq V_{a s}=0$ and $V[n, c] \subseteq V_{a s}=0$. Similarly, $[n, c] V=0$ and $[n, c] \subseteq$ Ann $V=0$. The contradiction shows that $A$ satisfies the identity $\left[[x, y]^{4}, z\right]=0$. Since $A$ is prime, the Posner theorem (see [14]) implies that $A$ is a central order in a generalized quaternion algebra.

Therefore, $Z(A) \neq 0$ and the algebra of quotients $\left(Z^{*}\right)^{-1} A$ is a generalized quaternion algebra $\mathbb{H}$ or a Cayley-Dickson algebra $\mathbb{O}$ over $\left(Z^{*}\right)^{-1} Z$. Let us show that $V$ is a $\left(Z^{*}\right)^{-1} A$-bimodule.

Lemma 4.1. Let $A$ be a prime superalgebra, $Z=Z(A)_{\overline{0}} \neq 0$. Then $\lambda(z)=\rho(z) \in$ $\mathcal{Z}(V)$ for any $z \in Z$ and $V$ is an irreducible faithful $\left(Z^{*}\right)^{-1} A$-bimodule.

Proof. By Lemma 3.1. for any $z \in Z, a \in A$ the set $(V, a, z)$ is an $A$-subbimodule of $V$. If $(V, a, z)=V$, then, by (44) we have

$$
V=(V, a, z)=((V, a, z), a, z)=0,
$$

a contradiction. Hence $(V, a, z)=0$, and we have

$$
\begin{gathered}
(V z) a=V(z a)=V(a z)=(V a) z \subseteq V z, \\
a(V z)=(a V) z \subseteq V z,
\end{gathered}
$$

which proves that $V z$ is an $A$-subbimodule of $V$. Since $V$ is faithful, we have $V z=V$ for any non-zero $z \in Z$. Moreover, Ann $z$ is an $A$-subbimodule of $V$ too. Hence Ann $z=0$. In other words, the map $\rho(z) \in$ End $V$ is invertible.

We proceed to show that $[V, z]=0$. By (11), the set $[V, z]$ is an $A$-subbimodule of $V$. If $[V, z]=V$, then, by (2), for any $a, b \in A$,

$$
(V, a, b)=([V, z], a, b) \subseteq(a b, V, z)+a(b, V, z)+(a, V, z) b=0,
$$

a contradiction. Thus $[V, z]=0, \rho(z)=\lambda(z) \in \mathcal{Z}(V)$ and $V$ has a structure of a vector space over the field $\left(Z^{*}\right)^{-1} Z$. Clearly, $V$ also has a natural structure of a $\left(Z^{*}\right)^{-1} A$-bimodule, which is evidently faithful and irreducible. 
We now return to the proof of Theorem 4.1. If $\left(Z^{*}\right)^{-1} A=\mathbb{O}$, a Cayley-Dickson algebra, then $V \cong \operatorname{Reg} \mathbb{O}$, the regular bimodule [8]. Consider an element $m \in V$ corresponding to the identity 1 of $\mathbb{O}$; then $V=m \cdot \mathbb{O}$. On the other hand, $m \cdot A$ is a non-zero $A$-subbimodule of $V$. Hence $m \cdot A=V=m \cdot \mathbb{O}$. Consequently, for every $z \in Z$ there exists $a \in A$ such that $m \cdot z^{-1}=m \cdot a$. We have $m \cdot(1-a z)=0$. Therefore $1=a z$ and $z^{-1}=a$. Thus $A=\mathbb{O}$ is a Cayley-Dickson algebra.

If $\left(Z^{*}\right)^{-1} A=\mathbb{H}$, a generalized quaternion algebra, then $V$ is the Cayley bimodule Cay $L$, that is, $V$ is isomorphic to the left irreducible associative $\mathbb{H}$-module $L$ on which $\mathbb{H}$ acts as follows: $v \cdot a=a v$ and $a \cdot v=\bar{a} v$, where $a v$ is the associative action of $\mathbb{H}$ on $L$ and $a \mapsto \bar{a}$ is the symplectic involution in $\mathbb{H}$. In this case, consider $I=\operatorname{Ann}_{l} L:=\{a \in A \mid a L=0\}$. Obviously, $I$ is an ideal of $A$ and $V \cdot I=0$. Hence $(A, V, I)=0$. Using the Moufang identities, we obtain that $(V, A, A) I=I(V, A, A)=0$. Note that, by (2), $(V, A, A)$ is an $A$-subbimodule of $V$. It follows that $I=0$, because $V$ is not associative. Thus $L$ is a faithful irreducible left $A$-module. Therefore, $A$ is a primitive $P I$-algebra and, since $A$ can be embedded into $\mathbb{H}, A$ is a $P I$-algebra. By Kaplansky's Theorem, it follows that the center of $A$ is a field. Hence $A=\left(Z^{*}\right)^{-1} A=\mathbb{H}$.

\section{THE CASE $A_{\overline{1}}^{2}=0, A_{\overline{1}} \neq 0$}

5.1. $A=F 1 \oplus F x, x^{2}=\lambda$. We will first consider the minimal possible case when $\operatorname{dim}_{F} A_{\overline{1}}=1, A_{\overline{1}}=F x, x^{2}=0$. The category of alternative bimodules over the superalgebra $A=A_{\overline{1}}=F x$ is isomorphic to the category of unital alternative bimodules over the superalgebra $A^{\sharp}=F 1 \oplus F x$, therefore we will study the latter. In fact, in order to also include Pisarenko's result on bimodules over $F[\sqrt{1}]$, we will consider the more general case when $A=F 1 \oplus F x, x^{2}=\lambda \in F$.

Recall the definition of the universal multiplicative enveloping superalgebra $\mathcal{U}(A)$ of an alternative superalgebra $A$. Let $\bar{A}$ be an isomorphic copy of the vector space $A$ under the isomorphism $a \mapsto \bar{a}$. Consider the vector space direct sum $A \oplus \bar{A}$ with the $\mathbb{Z}_{2}$-grading induced by that on $A$. Then the tensor algebra $T(A \oplus \bar{A})$ has a natural structure of an associative superalgebra. Denote by $I_{\text {alt }}$ the ideal of $T(A \oplus \bar{A})$ generated by the set of elements

$$
\begin{aligned}
& a \otimes b-a b+(-1)^{|a||b|}(b \otimes a-b a), \\
& \bar{a} \otimes \bar{b}-\overline{a b}+(-1)^{|a||b|}(\bar{b} \otimes \bar{a}-\overline{b a}), \\
& a b-a \otimes b-\bar{a} \otimes b+(-1)^{|a||b|} b \otimes \bar{a}, \\
& \overline{a b}+\bar{a} \otimes b-(-1)^{|a||b|}(\bar{b} \otimes \bar{a}+b \otimes \bar{a}), \\
& c \otimes c-c^{2},
\end{aligned}
$$

where $a, b \in A_{\overline{0}} \cup A_{\overline{1}}, c \in A_{\overline{0}}$; the elements of the last type are needed only in the case of char $F=2$. Since the generators of the ideal $I_{\text {alt }}$ are homogeneous, the quotient algebra $\mathcal{U}(A):=T(A \oplus \bar{A}) / I_{\text {alt }}$ inherits the superalgebra structure from $T(A)$.

Consider a pair of linear mapping $(\mathcal{L}, \mathcal{R}): A \rightarrow \mathcal{U}(A)$ defined as follows: $\mathcal{L}: a \mapsto$ $\mathcal{L}_{a}:=\bar{a}+I_{\text {alt }}, \mathcal{R}: a \mapsto \mathcal{R}_{a}:=a+I_{\text {alt }}$; then for any alternative birepresentation $(\lambda, \rho): A \rightarrow$ End $V$ there exists a unique (super)algebra homomorphism $\varphi: \mathcal{U}(A) \rightarrow$ End $V$ such that $\lambda=\varphi \circ \mathcal{L}, \rho=\varphi \circ \mathcal{R}$. Conversely, every homomorphism $\varphi: \mathcal{U}(A) \rightarrow$ End $V$ defines a structure of an alternative $A$-bimodule on $V$. In other words, the 
categories $\operatorname{Bimod}_{\mathrm{Alt}}-A$ and $\operatorname{Mod}_{\mathrm{As}} \mathcal{U}(A)$ are isomorphic. The (super)algebra $\mathcal{U}(A)$ is called the universal multiplicative enveloping (super)algebra of $A$.

If $A$ has a unit 1 , then the quotient algebra $\mathcal{U}_{1}(A):=\mathcal{U}(A) /\left(1-\mathcal{R}_{1}, 1-\mathcal{L}_{1}\right)$ is called the universal unital multiplicative enveloping (super)algebra of $A$.

Proposition 5.1. Let $A=F 1 \oplus F x, x^{2}=\lambda, A_{\overline{0}}=F 1, A_{\overline{1}}=F x$. Then the universal unital multiplicative enveloping superalgebra $\mathcal{U}_{1}(A)$ is a free 4-dimensional module over its center which is isomorphic to the polynomial ring $F[t]$. More exactly, it has a basis $\{1, a, b, a b\}$ over $F[t]$, where $a^{2}=t, b^{2}=t-2 \lambda, a \circ b=\lambda-t$.

If $\operatorname{char} F=3$ and $\lambda=0$, then $\mathcal{U}_{1}(A)$ has a graded ideal

$$
I:=F[t](a b-t-\lambda)+F[t](a-b)
$$

such that $I^{2}=0$, and in this case $\mathcal{U}_{1}(A) / I \cong(F[t])[\sqrt{t}]$.

If char $F \neq 3$ or $\lambda \neq 0$, then $\mathcal{U}_{1}(A)$ is a prime superalgebra whose central closure is a generalized quaternion algebra over the field $F(t)$.

Proof. Denote $a:=\mathcal{R}_{x}, b:=\mathcal{L}_{x}$; then $\mathcal{U}_{1}(A)=\operatorname{alg}\langle 1, a, b\rangle$. It follows from (11) that the elements $a, b$ satisfy the relations

$$
a^{2}-\lambda=b^{2}+\lambda=-a \circ b .
$$

Denote $t:=a^{2}$; then $t$ is an even central element of $\mathcal{U}_{1}(A)$, and one can easily check that $\mathcal{U}_{1}(A)$ is spanned as an $F[t]$-module by the elements $1, a, b, a b$. Moreover, a standard use of the Gröbner-Shirshov basis method [2 4] (known also as The Diamond Lemma) shows that $\mathcal{U}_{1}(A)$ is a free $F[t]$-module and $F[t]$ is a polynomial ring.

If char $F=3$ and $\lambda=0$, then one can easily check that $I$ is an ideal in $\mathcal{U}_{1}(A)$ and $I^{2}=0$. The quotient superalgebra $\overline{\mathcal{U}}:=\mathcal{U}_{1}(A) / I$ has the form $\overline{\mathcal{U}}=F[t] \oplus$ $F[t] \bar{a}, \bar{a}^{2}=t$, where $\overline{\mathcal{U}}_{\overline{0}}=F[t], \overline{\mathcal{U}}_{\overline{1}}=F[t] \bar{a}$.

Now let $\operatorname{char} F \neq 3$ or $\lambda \neq 0$. Since $\mathcal{U}_{1}(A)$ is a free module over the center $F[t]$, we may consider the central closure $\tilde{\mathcal{U}}:=(F[t])^{-1} \mathcal{U}_{1}(A)$ which has the same basis $\{1, a, b, a b\}$ over the field $F(t)$. Denote $u:=-\frac{1}{t-\lambda} a b$; then $u^{2}=u-\frac{t(t-2 \lambda)}{(t-\lambda)^{2}}$ and the subalgebra $F(t)[u]$ has a structure of a composition algebra over $F(t)$ with the involution $u \mapsto \bar{u}=1-u$. One can easily check that $\tilde{\mathcal{U}} \cong(F(t)[u], t)$, that is, $\tilde{\mathcal{U}}$ is obtained from $F(t)[u]$ by the Cayley-Dickson process with the parameter $t$ [30, section 2.2]. Hence $\tilde{\mathcal{U}}$ is a generalized quaternion algebra.

We can now classify irreducible bimodules over the superalgebra

$$
A=F 1 \oplus F x, x^{2}=\lambda .
$$

Theorem 5.1. Let $V$ be a unital irreducible faithful alternative non-associative bimodule over the superalgebra $A=F 1 \oplus F x, x^{2}=\lambda$. Then there exists a simple algebraic field extension $K:=F(\alpha), \alpha \neq \lambda$, such that $V$ is a $K$-vector space of $\operatorname{dim}_{K} V \leq 4$, and up to the changing of parity we have the following possibilities.

If the polynomial $f(u):=u^{2}+(\alpha-\lambda) u+\alpha(\alpha-2 \lambda)$ has a root $\varepsilon \neq 0$ in $K$, then $V=K v \oplus K v x, V_{\overline{0}}=K v, V_{\overline{1}}=K v x$, and the action of $A$ is given as follows:

$$
\begin{aligned}
& V_{\alpha, \lambda}^{\varepsilon}(1 \mid 1): \text { if } \alpha \neq 0 \text {, then } \\
& \qquad \begin{aligned}
v \cdot x=v x, \quad x \cdot v=\frac{\varepsilon}{\alpha} v x, \\
v x \cdot x=\alpha v, \quad x \cdot v x=(\alpha+\varepsilon-\lambda) v ;
\end{aligned}
\end{aligned}
$$


$V_{\lambda}(1 \mid 1):$ if $\alpha=0$, then

$$
\begin{aligned}
& v \cdot x=v x, \quad x \cdot v=-2 v x, \\
& v x \cdot x=0, \quad x \cdot v x=-\lambda v .
\end{aligned}
$$

If the polynomial $f(u)$ above is irreducible over $K$, then $\mathcal{U}(\alpha):=\mathcal{U}_{1}(A) /(t-\alpha)$ is a graded division algebra over $K$, and $V$ is isomorphic to the left regular supermodule over $\mathcal{U}(\alpha)$. More exactly,

$V_{\alpha, \lambda}(2 \mid 2): \operatorname{dim}_{K} V=4, V$ has a basis $\{v, x v, v x, x(v x)\}, V_{\overline{0}}=K v \oplus$ $K x(v x), V_{\overline{1}}=K v x \oplus K x v$, and the action of $A$ is given as follows:

$$
\begin{aligned}
v \cdot x=v x, & x \cdot v=x v, \\
v x \cdot x=\alpha v, & x \cdot v x=x(v x), \\
x v \cdot x=x(v x)+(\lambda-\alpha) v, & x \cdot x v=(2 \lambda-\alpha) v, \\
x(v x) \cdot x=(\alpha-\lambda) v x+\alpha x v, & x \cdot x(v x)=(2 \lambda-\alpha) v x .
\end{aligned}
$$

Conversely, for any simple algebraic field extension $K:=F(\alpha), \alpha \neq \lambda$, the above defined superbimodules $V_{\alpha, \lambda}^{\varepsilon}(1 \mid 1)$ and $V_{\alpha, \lambda}(2 \mid 2)$ are irreducible and alternative.

Proof. Notice first that every irreducible alternative bimodule $V$ over a unital (super)algebra is necessarily unital. In fact, by Lemma 4.1 $\lambda(1)=\rho(1) \in \mathcal{Z}(V)$, which implies that $W:=\{v-v \cdot 1 \mid v \in V\}$ is a subbimodule of $V$. Moreover, $W \neq V$ since $1 \in$ Ann $W$ and Ann $V=0$. Thus $W=0$, and $V$ is unital.

Therefore, the bimodule $V$ in the theorem is a unital associative irreducible $\mathcal{U}$ module for $\mathcal{U}:=\mathcal{U}_{1}(A)$. Let $\varphi: \mathcal{U} \rightarrow$ End $V$ be the representation of $\mathcal{U}$ corresponding to the $\mathcal{U}$-supermodule $V$; then $\varphi(t)=\alpha$ lies in the even part of the centralizer of $V$ which is a graded division algebra. Thus we may consider $\varphi(\mathcal{U}) \subseteq$ End $V$ as a homomorphic image of the superalgebra $\mathcal{U}(\alpha)=\mathcal{U} /(t-\alpha)$ over the field $K=F(\alpha)$ with the same basis $\{1, a, b, a b\}$, where $a^{2}=\alpha, b^{2}=\alpha-2 \lambda, a \circ b=\lambda-\alpha$. It is easy to see that if $\alpha=\lambda$, then $V$ is associative. Thus $\alpha-\lambda \neq 0$. Furthermore, since $\varphi(\mathcal{U})$ is finitely generated over $F$ and finite-dimensional over $K=F(\alpha)$, it is easy to see that $\alpha$ is algebraic over $F$ (see, for example, [12, Lemma 1.1]).

If char $F=3$ and $\lambda=0$, then by Proposition 5.1 the superalgebra $\mathcal{U}(\alpha)$ has the ideal $I(\alpha)$ with $I(\alpha)^{2}=0$. Since $V$ is irreducible, $\varphi(I)=0$, thus $\varphi(\mathcal{U}) \cong$ $\mathcal{U}(\alpha) / I(\alpha)=K \oplus K \bar{a}, \bar{a}^{2}=\alpha$. It is clear that in this case $V$ as a $\varphi(\mathcal{U})$-module is isomorphic to the regular supermodule, which coincides with the bimodule $V_{\alpha, \lambda}^{\varepsilon}(1 \mid 1)$ for $\varepsilon=\alpha$ and $\lambda=0$.

Let char $F \neq 3$ or $\lambda \neq 0$; then the superalgebra $\mathcal{U}(\alpha)$ is simple and isomorphic to a generalized quaternion superalgebra over $K$, with $\mathcal{U}(\alpha)_{\overline{0}}=K 1+K a b, \mathcal{U}(\alpha)_{\overline{1}}=$ $K a+K b$. Therefore, in this case $\varphi(\mathcal{U})=\mathcal{U}(\alpha)$, and we have the two possibilities, according to the structure of $\mathcal{U}(\alpha)_{\overline{0}}$.

If $\mathcal{U}(\alpha)_{\overline{0}}$ is a field, that is, the polynomial $f(u)=u^{2}+(\alpha-\lambda) u+\alpha(\alpha-2 \lambda)$ is irreducible over $K$, then $V \cong \operatorname{Reg} \mathcal{U}(\alpha)$ as a right $\mathcal{U}(\alpha)$-supermodule, and $V \cong$ $V_{\alpha, \lambda}(2 \mid 2)$ as an $A$-bimodule.

If the polynomial $f(u)$ has a root $\varepsilon$ in $K$, then the $K$-subspace

$$
L:=\operatorname{vect}\langle a b-\varepsilon,(\lambda-\alpha-\varepsilon) a-\alpha b\rangle
$$

is a minimal graded right ideal of $\mathcal{U}(\alpha)$. It produces the irreducible superbimodule $V_{\alpha, \lambda}^{\varepsilon}(1 \mid 1)$. Since $\tilde{\mathcal{U}}$ is simple and finite-dimensional, any other irreducible graded right $\mathcal{U}(\alpha)$-module up to the changing of parity is isomorphic to $L$. 
Remark 5.1. Observe that we have the following isomorphisms:

$$
V_{\alpha, \lambda}^{\varepsilon_{1}}(1 \mid 1)^{\mathrm{op}} \cong V_{\alpha, \lambda}^{\varepsilon_{2}}(1 \mid 1), \quad V_{\alpha}(2 \mid 2)^{\mathrm{op}} \cong V_{\alpha}(2 \mid 2),
$$

where $f(u)=\left(u-\varepsilon_{1}\right)\left(u-\varepsilon_{2}\right)$.

Remark 5.2. If the field $F$ is algebraically closed, then we have $K=F ; \lambda \in\{0,1\}$, $f(u)$ reducible and $\operatorname{dim}_{F} V=2$. Moreover, if $\lambda=0$, we may take $\alpha=1$, and for $\alpha=0$ we may take $\lambda=1$. Thus, up to the changing of parity, $V=V_{1}(1 \mid 1), V=$ $V_{\alpha, 1}^{\varepsilon}(1 \mid 1)$, or $V=V_{1,0}^{\varepsilon}(1 \mid 1)$, where in the last case $\varepsilon^{2}+\varepsilon+1=0$.

For $\lambda=1$ observe also that the bimodules $V_{1}(1 \mid 1), V_{2,1}^{0}(1 \mid 1)$ are isomorphic to the bimodules of type 1,2 from Theorem [2.2. and $V_{\alpha, 1}^{\varepsilon}(1 \mid 1)$ is isomorphic to the bimodule of type 3 for the pair $(\alpha-1, \varepsilon)$.

Remark 5.3. In the case when $A=A_{1}=F x, x^{2}=0$, we have the isomorphism $\mathcal{U}(A) \cong \mathcal{U}_{1}\left(A^{\sharp}\right)$, hence $A$ has the same irreducible superbimodules as the $A^{\sharp}$ does.

5.2. $A_{\overline{1}}^{2}=0$, the general case. We will consider now the general case of superalgebras with $A_{1}^{2}=0$.

Theorem 5.2. Let $V$ be an irreducible faithful alternative bimodule over the superalgebra $A$ with $A_{\overline{1}}^{2}=0$. Then there exist a field extension $K$ of $F$, a non-zero algebraic element $\alpha \in K$, an $F$-subspace $T$ and an $F$-subalgebra $S$ of $K$ such that:

i) $V$ is a unital irreducible faithful alternative bimodule over the superalgebra $B:=K \oplus K u, u^{2}=0$, and the structure of $V$ as a B-bimodule is given by Theorem [5.1, with $\rho(u)^{2}=\alpha$.

ii) $S \oplus T u$ is an $F$-subsuperalgebra of $B$ which is isomorphic to $A$.

iii) $K=\operatorname{alg}_{F}\left\langle\alpha T^{2}\right\rangle$.

$i v)$ The action of $A$ on $V$ is inherited by that of $B$.

Conversely, let $K$ be a field extension of $F$, and let $T, S$ and $\alpha$ be an $F$-subspace, $F$ subalgebra and $F$-algebraic element of $K$ such that $S T+T S \subseteq T$ and $\operatorname{alg}_{F}\left\langle\alpha T^{2}\right\rangle=$ $K$. Consider the superalgebra $B:=K \oplus K u, u^{2}=0$; then $A=S \oplus T u$ is a subsuperalgebra of $B$ and every irreducible faithful alternative birepresentation $(\lambda, \rho)$ of $B$ with $\rho(u)^{2}=\alpha$ is so over $A$.

Proof. Consider the subsuperalgebra $\Gamma$ of the centroid $\mathcal{E}=\mathcal{E}(E)$ of the split null extension $E=V \oplus A: \Gamma=\{\alpha \in \mathcal{E} \mid V \alpha \subseteq V, A \alpha \subseteq A\}$. By Proposition 3.1 $\Gamma$ is supercommutative and isomorphic to a subsuperalgebra of the centralizer $\mathcal{Z}=$ $\mathcal{Z}(V)$ which is a division superalgebra. We will identify $\Gamma$ with its image in $\mathcal{Z}$.

Let $(L, R): A \rightarrow$ End $E$ be the regular birepresentation of $A$ in $E$; then for any $x, y \in A_{\overline{1}}$ the operator $D:=R_{x} R_{y}$ is a derivation of the superalgebra $E$ due to (2). Since $\left.D\right|_{A}=0$, it follows that $D \in \Gamma \subseteq \mathcal{Z}$.

If $R_{x} R_{y}=0$ for all $x, y \in A_{\overline{1}}$, then $\left(V A_{\overline{1}}\right) A_{\overline{1}}=0$. But $V A_{\overline{1}}$ is a subbimodule of $V$, and if $V=V A_{\overline{1}}$, then $V=\left(V A_{\overline{1}}\right) A_{\overline{1}}=0$, a contradiction. Thus $V A_{\overline{1}}=0$. It follows from (11) that $L_{x} L_{y}=R_{x} R_{y}=0$, hence we have $A_{\overline{1}}\left(A_{\overline{1}} V\right)=0$ and $A_{\overline{1}} V=0$ as well. Thus $A_{\overline{1}} \subseteq$ Ann $V=0$, a contradiction.

Let $R_{x} R_{y} \neq 0$, show that then $R_{x}^{2} \neq 0$. In fact, we have seen that $0 \neq D=$ $R_{x} R_{y} \in \mathcal{Z}$. Therefore, $V=V D=V D^{2}=V R_{x} R_{y} R_{x} R_{y}$. But $R_{x} R_{y}=R_{y} R_{x}$ by (11), hence $V=V R_{x}^{2} R_{y}^{2}$ and $R_{x}^{2} \neq 0$. Since $R_{x}^{2}$ is invertible on $V$, so is $R_{x}$.

Assume now that $R_{z} R_{t}=0$ for some $z, t \in A_{\overline{1}}$; then $R_{t}$ is not invertible and hence $R_{u} R_{t}=L_{u} L_{t}=0$ for any $u \in A_{\overline{1}}$. Thus $V A_{\overline{1}} R_{t}=0=\left(A_{\overline{1}} V\right) L_{t}$ and $t=0$. 
We proved that for any $0 \neq x \in A_{\overline{1}}$ the operator $R_{x}$ is invertible on $V$. In particular, we have $V_{\overline{1}}=V_{\overline{0}} x, V_{\overline{0}}=V_{\overline{1}} x$.

Below let $v \in V, x, y \in A_{\overline{1}}, a, b, c \in A_{\overline{0}}$. From super-linearized identity (10) we have

$$
(v x)(a y) \pm(y x)(a v)=(v(x a)) y \pm(y(x a)) v
$$

which gives $(v x)(a y)=(v(x a)) y$ or

$$
R_{x} R_{a y}=R_{x a} R_{y} .
$$

For $y=x$ we have $R_{x} R_{a x}=R_{x} R_{x a}$, which by invertibility of $R_{x}$ implies $R_{x a}=R_{a x}$ or $R_{[a, x]}=0$, and eventually $[a, x]=0$, that is,

$$
\left[A_{\overline{0}}, A_{\overline{1}}\right]=0 \text {. }
$$

Furthermore, applying (12) and (13), we get

$$
R_{(a x) b} R_{x}=R_{a x} R_{b x}=R_{a x} R_{x b}=R_{x b} R_{a x}=R_{(x b) a} R_{x}=R_{a(x b)} R_{x}
$$

which implies $R_{(a, x, b)}=0$ and therefore

$$
\left(A_{\overline{0}}, A_{\overline{1}}, A_{\overline{0}}\right)=0 \text {. }
$$

Let $R$ be the $F$-subalgebra of $\mathcal{Z}_{0}$ generated by the elements $\left\{R_{x} R_{y} \mid x, y \in A_{\overline{1}}\right\}$; then $R \subseteq \Gamma_{\overline{0}}$ is a commutative domain. Denote by $K$ the field of fractions of $R$. Fix $0 \neq x \in A_{\overline{1}}$ and let $\alpha:=\left(R_{x}\right)^{2}$. Then for any $y \in A_{\overline{1}}, R_{y}=\left(R_{x} R_{y}\right) \alpha^{-1} R_{x}$. Consider the $F$-linear mapping $\tau: A_{\overline{1}} \rightarrow K$ given by $\tau(y)=\left(R_{x} R_{y}\right) \alpha^{-1}$; then $R_{y}=\tau(y) R_{x}$. Clearly, $\operatorname{ker} \tau=0$, hence $T=\tau\left(A_{\overline{1}}\right)$ is an $F$-vector subspace of $K$ isomorphic to $A_{\overline{1}}$. Furthermore, define an $F$-linear mapping $\sigma: A_{\overline{0}} \rightarrow K$ by $\sigma(a)=\tau(a x)$ and denote $S:=\sigma\left(A_{\overline{0}}\right)$.

Lemma 5.1. Let $B=K \oplus K u, u^{2}=0$, with $B_{\overline{0}}=K, B_{\overline{1}}=K u$; then the map $\varphi: a+y \mapsto \sigma(a)+\tau(y) u$ is an isomorphism of $A$ and $S \oplus T u \subseteq B$. In particular, $A_{\overline{0}}$ is an associative and commutative domain.

Proof. Let us prove first that $\varphi$ is a homomorphism. It holds if $\tau$ and $\sigma$ satisfy the conditions:

$$
\tau(a y)=\sigma(a) \tau(y), \sigma(a b)=\sigma(a) \sigma(b),
$$

for all $a, b \in A_{\overline{0}}, y \in A_{\overline{1}}$.

We have

$$
\begin{aligned}
\sigma(a) \tau(y) & =\left(R_{x} R_{a x} \alpha^{-1}\right)\left(R_{x} R_{y} \alpha^{-1}\right)=R_{a x} R_{y} R_{x} R_{x} \alpha^{-2} \\
& =R_{a x} R_{y} \alpha^{-1} \stackrel{12}{=} R_{x} R_{a y} \alpha^{-1}=\tau(a y)
\end{aligned}
$$

proving the first equality. Furthermore,

$$
\begin{aligned}
& \sigma(a) \sigma(b)=\tau(a x) \tau(b x)=\left(R_{x} R_{a x} \alpha^{-1}\right)\left(R_{x} R_{b x} \alpha^{-1}\right)=R_{a x} R_{b x} \alpha^{-1} \\
& \stackrel{12}{=} R_{x} R_{a(b x)} \alpha^{-1} \stackrel{14}{=} R_{x} R_{(a b) x)} \alpha^{-1}=\sigma(a b) \text {, }
\end{aligned}
$$

which proves the second equality. Therefore, $\varphi$ is a homomorphism. We have already noted that $\operatorname{ker} \tau=0$. Now let $a \in \operatorname{ker} \sigma$; then $\tau(a y)=\sigma(a) \tau(y)=0$ for any $y \in A_{\overline{1}}$ and $a A_{\overline{1}}=0$. By Lemma 3.1, in view of (13) and (14), $(V, a, x)$ is a subbimodule of $V$. If $(V, a, x)=0$, then we have $R_{a} R_{x}=0$ and $R_{a}=0$. Similarly, $L_{a}=0$ and $a \in \operatorname{Ann} V=0$. If $(V, a, x)=V$, then $V a=(V, a, a x)=0$ and $a V=0$, and again $a=0$. Therefore, $\operatorname{ker} \sigma=0$, and $\varphi$ is an isomorphism. 
Lemma 5.2. For any $a \in A_{\overline{0}}, y \in A_{\overline{1}}, s \in A_{\overline{0}} \cup A_{\overline{1}}$ hold

$$
\begin{aligned}
\text { i) } \quad R_{a}=L_{a}=\sigma(a), & \text { ii) } R_{a} R_{s}=R_{a s}, \\
\text { iii) } \quad R_{y}=\tau(y) R_{x}, & \text { iv) } L_{y}=\tau(y) L_{x} .
\end{aligned}
$$

Proof. By Lemma 3.1. $(V, a, b)$ is a subbimodule of $V$. If it is not zero, then by (4) we have $V=(V, a, b)=((V, a, b), a, b)=[a, b](V, a, b)=0$, a contradiction. Therefore $R_{a} R_{b}=R_{a b}=R_{b a}=R_{b} R_{a}$.

We have already seen that $(V, a, x)$ is a subbimodule of $V$ as well for any $x \in A_{\overline{1}}$. If $(V, a, x)=0$, then $R_{a} R_{x}=R_{a x}=R_{x a}=R_{x} R_{a}$. Moreover, in this case we have $\sigma(a) R_{x}=R_{a x}=R_{a} R_{x}$, implying $R_{a}=\sigma(a)$. Similarly, $L_{a}=\sigma(a)$, hence $\left.i\right)$ is true.

Assume that $(V, a, x)=V$. Then for any $v \in V$ we have

$$
(v, a, x) a=(v, a, a x)=(v, a, x a)=a(v, a, x),
$$

that is, $R_{a}=L_{a}$. Furthermore, we have $R_{a} \circ R_{x}=2 R_{a x},\left[R_{a}, R_{x}\right]=\left[L_{a}, R_{x}\right]=$ $-R_{a} R_{x}+R_{a x}$. Summing the two equations we get $3 R_{a} R_{x}=3 R_{a x}$. By (2), $R_{a} R_{x}-$ $R_{a x} \in \Gamma_{\overline{1}} \subseteq \mathcal{Z}_{\overline{1}}$. Since $\Gamma$ is a supercommutative domain, $\Gamma_{\overline{1}}$ may be non-zero only if char $F=2$. Hence we have $R_{a} R_{x}=R_{a x}$, and as above $R_{a}=L_{a}=\sigma(a)$.

We have already seen that $R_{y}=\tau(y) R_{x}$. To prove $i v$ ), consider

$$
\begin{aligned}
L_{y} & =L_{y} \alpha \alpha^{-1}=L_{y} R_{x} R_{x} \alpha^{-1}=L_{y} L_{x} L_{x} \alpha^{-1} \\
& =R_{y} R_{x} L_{x} \alpha^{-1}=\tau(y) L_{x} .
\end{aligned}
$$

We now return to the proof of Theorem 5.2. Assume first that $L_{x} R_{x} \notin \mathcal{Z}$; then there exists $v \in V_{\overline{0}}$ such that $v$ and $w=v L_{x} R_{x}$ are linearly independent over the division algebra $\mathcal{Z}_{\overline{0}}$. For $y, z \in A_{\overline{1}}$ we have $L_{y} R_{z}=\left(R_{y} R_{z} \alpha^{-1}\right)\left(L_{x} R_{x}\right) \in$ $R\left[\alpha^{-1}\right] L_{x} R_{x}$. Since $V$ is irreducible, it follows from Lemma 5.2 and (11) that $v R+w R\left[\alpha^{-1}\right]=V_{\overline{0}}$. Thus we have

$$
V_{\overline{0}}=v R+w R\left[\alpha^{-1}\right] \subseteq v K+w K \subseteq v \mathcal{Z}_{\overline{0}}+w \mathcal{Z}_{\overline{0}} \subseteq V_{\overline{0}},
$$

which implies that $R=K=\mathcal{Z}_{\overline{0}}$.

Assume now that $L_{x} R_{x} \in \mathcal{Z}$; then we have $L_{x} R_{x} R_{x}=R_{x} L_{x} R_{x}$ which implies $L_{x} R_{x}=R_{x} L_{x}$. By (11), $2 L_{x} R_{x}=\left[L_{x}, R_{x}\right]_{s}=-\left(R_{x}\right)^{2}=-\left(L_{x}\right)^{2}$. Therefore, $2 L_{x}=-R_{x}, 2 R_{x}=-L_{x}$, implying $L_{x}=R_{x}$. Now for any $0 \neq v \in V_{\overline{0}}$ we have $V_{\overline{0}}=v R$, which implies as above $R=K=\mathcal{Z}_{\overline{0}}$. Notice that $R_{y} R_{z}=\tau(y) \tau(z) \alpha \in$ $T^{2} \alpha$, hence $K=\operatorname{alg}\left\langle T^{2} \alpha\right\rangle$.

Define a unital $K$-linear action of the superalgebra $B=K \oplus K u$ on $V$ by setting

$$
v \cdot u=v R_{x}, u \cdot v=v L_{x}
$$

then evidently $V$ is an alternative irreducible faithful $B$-bimodule. The restriction of this action on $S \oplus T u \cong A$ coincides with the $A$-bimodule structure of $V$.

Conversely, let $K$ be a field extension of $F$, and let $T, S$ and $\alpha$ be an $F$ subspace, $F$-subalgebra and $F$-algebraic element of $K$ such that $S T+T S \subseteq T$ and $\operatorname{alg}_{F}\left\langle\alpha T^{2}\right\rangle=K$. Consider the superalgebra $B=K \oplus K u, u^{2}=0$; then $A=S \oplus T u$ is a subsuperalgebra of $B$. Let $V$ be an irreducible unital faithful alternative $B$-superbimodule such that $\rho(u)^{2}=\alpha$, and assume that $W \subseteq V$ is an $A$-subsuperbimodule of $V$. We have for any $w \in W, t_{1}, t_{2} \in T:\left(w \cdot t_{1} u\right) \cdot t_{2} u=$ $\left(t_{1} t_{2}\right)(w \cdot u) \cdot u=\left(\alpha t_{1} t_{2}\right) w \in W$, hence $W$ is a $K$ subspace of $V$. Let $0 \neq t \in T$; then $W \supseteq t^{-1} W \cdot(t u)=W \cdot u$, and $W=V$. 
Remark 5.4. Observe that the case $L_{x}=R_{x}$ appears only when char $F=3$. It follows from Lemma 5.2 that in this case $L_{y}=R_{y}$ for arbitrary $y \in A_{\overline{1}}$. Thus $V$ is supercommutative, and we have

$$
3 v R_{x} R_{y}=3(v, x, y)=3(x, y, v) \stackrel{\underline{1}}{=}[x y, v]-x[y, v]-(-1)^{|y||v|}[x, v] y=0,
$$

which is impossible if char $F \neq 3$. Reciprocally, if char $F=3$, then $\left(L_{x} R_{x}-\alpha\right)^{2}=$ $-\alpha^{2}-\alpha L_{x} R_{x}-2 \alpha L_{x} R_{x}+\alpha^{2}=0$ (see Section 5.1). Therefore, $L_{x} R_{x}=\alpha=R_{x} R_{x}$, and $R_{x}=L_{x}, V$ is supercommutative.

\section{Bimodules OVER THE SIMPle SUPERALGEBRAS}

6.1. Reduction of prime superalgebras to simple ones. We start this section with reduction of the case of prime associative superalgebras to the simple finitedimensional ones.

We will say that a superalgebra $A$ with the center $Z(A)=Z_{\overline{0}} \oplus Z_{\overline{1}}$ is an even central order in a superalgebra $B$ if $B=\left(Z_{\overline{0}}^{*}\right)^{-1} A$. In this case we will also call $B$ the even central closure of $A$.

Lemma 6.1. Let $A$ be a prime associative superalgebra and let $V$ be a faithful irreducible alternative $A$-superbimodule which is not associative. Then $Z_{\overline{0}}=$ $Z(A)_{\overline{0}} \neq 0$, and the even central closure $B=\left(Z_{\overline{0}}^{*}\right)^{-1} A$ is a simple superalgebra which is finite-dimensional over the even part of its supercenter $\left(Z_{\overline{0}}^{*}\right)^{-1} Z$. Moreover, $\left(Z_{\overline{0}}^{*}\right)^{-1} Z \subseteq \mathcal{Z}(V)$, and $V$ has a natural structure of a B-bimodule.

Proof. Since $A$ is associative, we can repeat the arguments from Section 4 to get $V_{a s}:=\{v \in V \mid(v, A, A)=0\}=0$ and $V \cdot\left[N, A_{\overline{0}}\right]=0$, where $N$ is the associative center of the split null extension $E=A \oplus V$. Therefore $A_{\overline{0}}$ satisfies the identity $\left[[x, y]^{4}, z\right]=0$. By [1], $A$ is also a $P I$-algebra.

Consider the quotient algebra $A / \mathcal{B}(A)$, where $\mathcal{B}(A)$ is the Baer radical of the algebra $A$. Since $A / \mathcal{B}(A)$ is semiprime (as algebra) and $P I$, it follows that there exists a non-trivial central polynomial $g\left(x_{1}, \ldots, x_{n}\right)$ of $A / \mathcal{B}(A)\left[14\right.$. Hence $\left[g\left(a_{1}, \ldots, a_{n}\right), A\right]$ $\subseteq \mathcal{B}(A)$ for arbitrary homogeneous elements $a_{k}$. But the Baer radical of a prime superalgebra does not contain non-zero homogeneous elements (see M. Cohen and S. Montgomery [6]), therefore $\left[g\left(a_{1}, \ldots, a_{n}\right), A\right]=0$ and $Z_{\overline{0}} \neq 0$.

Consider the prime superalgebra $B=\left(Z_{\overline{0}}^{*}\right)^{-1} A$. Let $I$ be a non-zero graded ideal of $B$. Then $I$ is a prime superalgebra as well, and $I \cap Z_{\overline{0}}(B)=Z_{\overline{0}}(I) \neq 0$. The proof of these statements repeats verbatim the non-graded case. Hence $I=B$ and $B$ is a simple superalgebra. It is also clear that $Z_{\overline{0}}(B)=\left(Z_{\overline{0}}^{*}\right)^{-1} Z$ is a field.

Due to C. T. C. Wall [26], $B$ is simple as an algebra or there exists a proper (non-graded) ideal $I$ in $B$ such that $B=\pi_{0}(I) \oplus \pi_{1}(I)$, where $\pi_{i}: I \longrightarrow B_{i}$ are the natural projections. It is easy to see that in this case $B \cong B_{\overline{0}}[u]$ where $B_{\overline{0}}$ is simple.

In the first case, since $B$ is a simple $P I$-algebra it follows that $B$ is finitedimensional over $Z(B)$. But $Z(B)$ is finite-dimensional over $Z_{\overline{0}}(B)$. Indeed, if $Z_{\overline{1}}(B) \neq 0$, then, fixing $0 \neq z_{1} \in Z_{\overline{1}}(B)$, we have $z_{1}^{2}=\alpha \neq 0 \in Z_{\overline{0}}(B)$ and then for any $x \in Z_{\overline{1}}(B)$ we get $x=x\left(\alpha^{-1} z_{1}^{2}\right)=\left(\alpha^{-1} x z_{1}\right) z_{1} \in Z_{\overline{0}}(B) \cdot z_{1}$.

In the second case $B_{\overline{0}}$ is a simple $P I$-algebra, hence $B_{\overline{0}}$ is finite-dimensional over $Z\left(B_{\overline{0}}\right)$. It is easily seen that $B$ is finite-dimensional over $Z\left(B_{0}\right)$ too and, in this case, $Z(B)=Z\left(B_{\overline{0}}\right)[u]$. 
By Lemma 4.1, $Z(A)$ is contained in the centralizer $\mathcal{Z}(V)$ of the bimodule $V$, which is a graded division superalgebra. Therefore, $\left(Z_{\overline{0}}^{*}\right)^{-1} Z \subseteq \mathcal{Z}$ and $V$ has a natural structure of a $B$-bimodule. Clearly, $V$ is irreducible and faithful over $B$.

Let us call a prime alternative non-associative superalgebra $A$ of classical type if $A$ is an even central order in a simple non-trivial superalgebra $B$. Otherwise we will call $A$ exceptional. It follows from the classification of prime alternative superalgebras in [19] that if $A$ is exceptional, then necessarily char $A=3$, the even part $A_{\overline{0}}$ satisfies the identity $[x, y]^{4}=0$ and contains a non-zero nil-ideal $I$ such that $\left(I, A_{\overline{1}}, A_{\overline{1}}\right) \subseteq I$. It is still an open question whether exceptional superalgebras exist.

For prime superalgebras of classical type we have

Lemma 6.2. Let $A$ be a prime alternative superalgebra of classical type and let $V$ be a faithful irreducible alternative $A$-superbimodule. Then $Z_{\overline{0}}=Z(A)_{\overline{0}} \neq 0$, and the even central closure $B=\left(Z_{\overline{0}}^{*}\right)^{-1} A$ is a simple alternative superalgebra. As above, $\left(Z_{\overline{0}}^{*}\right)^{-1} Z \subseteq \mathcal{Z}(V)$, and $V$ has a natural structure of an irreducible B-bimodule.

Proof. The proof follows from Lemma 4.1 as above.

Therefore, except for the exceptional case of char 3, the classification of irreducible alternative non-associative superbimodules over prime superalgebras is reduced to bimodules over simple central finite-dimensional superalgebras, where we call a superalgebra $A$ over a field $F$ central if $Z(A)_{\overline{0}}=F$.

We will show next that, modulo the superalgebras of type $B=K[u]$, for the case of char $\neq 2$ it suffices to consider only non-associative superalgebras.

Lemma 6.3. In the conditions of Lemma 6.1, if char $F \neq 2$, then the even central closure $B$ of $A$ is of the type $B=K \oplus K s, s^{2}=\lambda \in K, \lambda \neq 0$, and the structure of $V$ and $A$ is given by Theorem 5.1 .

Proof. By Lemma 6.1, $Z=Z_{\overline{0}}(A) \neq 0, A$ is an even central order in a simple central superalgebra $B$ over the field $K=\left(Z_{\overline{0}}^{*}\right)^{-1} Z_{\overline{0}}$, and $V$ is a faithful irreducible alternative bimodule over $B$. Let $\tilde{K}$ be the algebraic closure of $K$; then $\tilde{B}=\tilde{K} \otimes B$ is a simple central superalgebra over $\tilde{K}$ and $\tilde{V}=\tilde{K} \otimes V$ is an alternative unital bimodule over $\tilde{B}$. If $\operatorname{dim}_{\tilde{K}} \tilde{B}>2$, then by [11] $\tilde{V}$ is completely reducible and all its irreducible components are associative. Hence in this case $V$ is associative, contrary to the assumption. Therefore, $\operatorname{dim}_{\tilde{K}} \tilde{B}=\operatorname{dim}_{K} B=2$ and $B=K \oplus K s, s^{2}=\lambda \in$ $K^{*}$.

Remark 6.1. Observe that, contrary to the case of algebras in Section 4, $A$ may be not simple and hence in general $B \neq A$. As an example, consider the superalgebra $B=\mathbb{Q}[\sqrt{1}]:=\mathbb{Q} \oplus \mathbb{Q} u, u^{2}=1$ and let $V:=\mathbb{Q} v_{0} \oplus \mathbb{Q} v_{1}$ be the irreducible supermodule of type 3 from Theorem 2.2 for $\alpha=-\frac{3}{7}, \delta=\frac{8}{7}$. Let $A=\mathbb{Q}_{7}[\sqrt{1}]:=$ $\mathbb{Q}_{7} \oplus \mathbb{Q}_{7} u, u^{2}=1$, where $\mathbb{Q}_{7}$ stands for the subring of rational numbers whose denominators are not divisible by 7 . Then $V$ is an irreducible faithful alternative $A$-bimodule, and $B=\left(Z_{\overline{0}}^{*}\right)^{-1} A \neq A$.

If one wanted $A$ to be an algebra over a field and not just a ring, then one may take in this example $A:=F[[x]][\sqrt{1}], B:=F((x))[\sqrt{1}], \alpha:=\frac{-x^{2}+1}{x^{2}+x+1}, \delta:=x(1+\alpha)$ where $F$ is a field, $F[[x]]$ and $F((x))$ are the algebra of formal series and the field of formal Laurent series over $F$. 
6.2. Superbimodules in characteristic 2. In this section, we will classify irreducible alternative non-associative superbimodules of characteristic 2 .

Recall that every $\mathbb{Z}_{2}$-graded alternative algebra of characteristic 2 is an alternative superalgebra. The simple superalgebras $\mathbb{O}(4 \mid 4)$ and $\mathbb{O}[u]$ provide us the irreducible superbimodules $\operatorname{Reg} \mathbb{O}(4 \mid 4), \operatorname{Reg}(\mathbb{O}[u])$.

Furthermore, consider a generalized quaternion algebra $\mathbb{H}$ with the $\mathbb{Z}_{2}$-grading coming from the Cayley-Dickson process; then it is an alternative superalgebra which we denote as $\mathbb{H}(2 \mid 2)$. As in the non-graded case, the category of $\mathbb{Z}_{2}$-graded Cayley bimodules over $\mathbb{H}(2 \mid 2)$ is isomorphic to the category of (graded) left $\mathbb{H}(2 \mid 2)$ modules. For a left $\mathbb{H}(2 \mid 2)$-module $L$ we again denote by Cay $L$ the corresponding Cayley $\mathbb{H}(2 \mid 2)$-bimodule. Then Cay $L$ is an alternative superbimodule over $\mathbb{H}(2 \mid 2)$ in the characteristic 2 case.

Finally, let $A$ be an alternative algebra and $V$ be an $A$-bimodule; consider the split null extension $E=E(A, V)$. The double $E[u]=E \oplus E u, u^{2}=\alpha \neq 0$ is an alternative superalgebra with the even part $E$ and the odd part $E u$ (recall that char $F=2$ ). Moreover, the ideal $V[u]=V \oplus V u$ may be considered as a bimodule over the alternative superalgebra $A[u]=A \oplus A u$, and if $V$ is irreducible, then so is $V[u]$. We will denote this bimodule as $V[u]$ or as $V[\sqrt{1}]$ when $u^{2}=1$.

Theorem 6.1. Let $V$ be an irreducible faithful alternative non-associative bimodule over a non-trivial prime alternative superalgebra $A$ of characteristic 2 with $\operatorname{dim}_{\left(Z^{*}\right)^{-1} Z}\left(Z^{*}\right)^{-1} A>2$. Then, up to the graded isomorphism, we have the following possibilities:

- $A=\mathbb{H}(2 \mid 2), V=$ Cay $L, L$ is a minimal $\mathbb{Z}_{2}$-graded left ideal of $\mathbb{H}(2 \mid 2)$;

- $A=\mathbb{H}[u], V=($ Cay $L)[u], L$ is a minimal left ideal of $\mathbb{H}$;

- $A=\mathbb{O}(4 \mid 4), V=\operatorname{Reg}(\mathbb{O}(4 \mid 4))$;

- $A=\mathbb{O}[u], V=\operatorname{Reg}(\mathbb{O}[u])$.

Proof. Consider first the associative case. By Lemma 6.1, $B=\left(Z^{*}\right)^{-1} A$ is a simple central superalgebra over the field $K=\left(Z^{*}\right)^{-1} Z$. Let $\tilde{K}$ be the algebraic closure of $K$; then $\tilde{B}=\tilde{K} \otimes_{K} B$ is a simple central superalgebra over $\tilde{K}$ and by C. T. C. Wall [26] $\tilde{B}$ is isomorphic to $M_{m \mid k}(\tilde{K}), 1 \leq k \leq m$, or to $M_{n}(\tilde{K})[\sqrt{1}], n>$ 1. Let us show that we necessarily have $m+k=n=2$. Consider $\tilde{V}:=\tilde{K} \otimes_{K} V$; it is a bimodule over $\tilde{B}$ and by $[30$, Lemma 7.5$]$ we have $[N, \tilde{B}]_{s} \subseteq \operatorname{Ann}(\tilde{V}, \tilde{B}, \tilde{B})$, where $N$ is the associative center of the split null extension $E(\tilde{B}, \tilde{V})$. Notice that since $B$ is associative and $V$ is irreducible, we have $V=(V, B, B)$. Therefore $V[N, \tilde{B}]_{s}=0$, which implies easily that $[N, \tilde{B}]_{s}=0$. In particular, the following inclusion holds in $\tilde{B}$ :

$$
\left[[a, x] \circ_{s}[a, y], a\right] \in Z_{\overline{0}}(\tilde{B})=\tilde{K}
$$

for any $a \in \tilde{B}_{\overline{0}}, x, y \in \tilde{B}_{\overline{1}}$. In fact, by [30. (7.25) and Lemma 7.1], we have the inclusion $\left[[a, b]^{2}, a\right] \in N(A)$ in any alternative algebra $A$. Superlinearizing this inclusion on $b$, we get the inclusion

$$
\left[[a, x] \circ_{s}[a, y], a\right] \in N(E),
$$

for any $a \in E_{\overline{0}}, x, y \in E_{\overline{0}} \cup E_{\overline{1}}$, which implies (15). Assume now that $\tilde{B}=M_{m \mid k}(\tilde{K})$ with $m>1$ and substitute in (15) $a=e_{11}+e_{22}+e_{12}, x=e_{1, m+1}, y=e_{m+1,1}$. 
We obtain

$$
\begin{aligned}
{\left[[a, x] \circ_{s}[a, y], a\right] } & =\left[\left[e_{1, m+1}, e_{m+1,1}+e_{m+1,2}\right], a\right] \\
& =\left[e_{11}+e_{12}+e_{m+1, m+1}, e_{11}+e_{22}+e_{12}\right]=e_{12} \notin \tilde{K},
\end{aligned}
$$

a contradiction. Similarly, assume that $\tilde{B}=M_{n}(\tilde{K})[\sqrt{1}]$ with $n>2$ and substitute in (15) $a=e_{11}+e_{22}+e_{12}, x=e_{13} u, y=e_{31} u$ :

$$
\begin{aligned}
{\left[[a, x] \circ_{s}[a, y], a\right] } & =\left[\left[u e_{13}, u\left(e_{31}+e_{32}\right)\right], a\right] \\
& =\left[e_{11}+e_{12}+e_{33}, e_{11}+e_{22}+e_{12}\right]=e_{12} \notin \tilde{K},
\end{aligned}
$$

a contradiction again.

Returning to the superalgebra $B$ we conclude that in the first case $B$ is a generalized quaternion superalgebra $\mathbb{H}(2 \mid 2)$ and in the second case $B_{\overline{0}} \cong \mathbb{H}$.

Let $B=\mathbb{H}(2 \mid 2), \quad B_{\overline{0}}=K \oplus K i, \quad B_{\overline{1}}=K j \oplus K k, i^{2}=i+\alpha, k=i j=$ $j(i+1), j^{2}=\beta$, where $\alpha, \beta \in K,(4 \alpha+1) \beta \neq 0$ (see [30, Chapter 2]). Substituting in (16) $a=i, x=j, y=v \in V$, we get

$$
\left(\left[[i, j] \circ_{s}[i, v], i\right], B, B\right)=([[[v, i], j], i], B, B)=0,
$$

that is, [[[V,i],j],i] $\subseteq V_{a s}=\{v \in V \mid(v, B, B)=0\}$ which is a subbimodule of $V$. Since $V$ is non-associative, $V_{a s}=0$, and we have $[[[V, i], j], i]=0$. Let $W:=\{w \in$ $V \mid[w, i]=0\}$, show that $W=0$. For any $w \in W, b \in B$ we have

$$
(w, b, i)=\left(w, b, i^{2}-\alpha\right)=\left(w, b, i^{2}\right)=(w \circ i, b, i)=([w, i], b, i)=0,
$$

that is, $(W, B, i)=0$. Further,

$$
(W, B, B) \circ i \subseteq(W \circ i, B, B)+W \circ(i, B, B)=0,
$$

thus $(W, B, B \circ i) \subseteq(W, B, B) \circ i+(W, B, i) \circ B=0$. Observe that $j=j \circ i, k=k \circ i$, which implies that $(W, B, B)=0$ and $W \subseteq V_{a s}=0$.

Therefore, we have $[[V, i], j]=0$ and similarly $[[V, i], k]=0$. Let us prove that $U=[V, i]=V \circ i$ is a subbimodule of $V$. Clearly, $U i+i U \subseteq U$. Note that $(V, B, i)=\left(V, B, i^{2}\right)=(V, B, i) \circ i \subseteq U$. We also have $[U, j]=[U, \bar{k}]=0$. Consider

$$
\begin{aligned}
{[v, i] j } & =\left[v, i^{2}\right] j=[v \circ i, i] j=[v \circ i, i j]-i[v \circ i, j]+3(v \circ i, i, j) \\
& =[v \circ i, k]+(v \circ i, i, j)=(v \circ i, i, j) \in(V, B, i) \subseteq U .
\end{aligned}
$$

Thus $U j \subseteq U$ and similarly $U k \subseteq U$, hence $U$ is a subbimodule of $V$. We have already showed that the mapping $v \mapsto v \circ i$ is injective. Therefore $U=V$, and we have $[V, j]=[V, k]=0$. Consider $3(v, j, j)=[v j, j]-v[j, j]+[v, j] j=0$. Similarly, we have $(V, k, k)=0$, which proves that $V$ is an alternative $\mathbb{Z}_{2}$-graded $B$-bimodule.

Denote $T:=\{v-[v, i] \mid v \in V\}$; then clearly $T i+i T \subseteq T$. Furthermore,

$$
\begin{aligned}
{[v, i] j } & =[v, i j]-i[v, j]+3(v, i, j)=3(v, i, j) \\
& =[v i, j]-v[i, j]-[v, j] i=v[i, j]=v j,
\end{aligned}
$$

and similarly $[v, i] k=v k$. Thus $T j=T k=0$ and $T$ is a subbimodule of $V$. Since $V$ is faithful, $T=0$ and we have $v \circ i=v$ for any $v \in V$. Therefore, $b v=v \bar{b}$ for any $b \in B$, and $V$ is a Cayley bimodule over $B$. Since $V$ is irreducible, $V=$ Cay $L$, where $L$ is an irreducible left $\mathbb{H}(2 \mid 2)$-module. It is isomorphic to a minimal graded left ideal of $B=\mathbb{H}(2 \mid 2)$. As at the end of the proof of Theorem 4.1, we get that $L$ is an irreducible graded left $A$-module and $A$ is a graded primitive $P I$-algebra. By the graded version of Kaplansky's theorem, $A$ is graded simple. Hence $Z(A)$ is a graded division algebra, $K=Z_{\overline{0}}$ and $A=B=\mathbb{H}(2 \mid 2)$. 
Consider now the case when $\tilde{B}=M_{2}(\tilde{K})[\sqrt{1}]=M_{2}(\tilde{K}) \oplus M_{2}(\tilde{K}) u$. We have $Z_{\overline{1}}(\tilde{B})=\tilde{K} u \neq 0$, therefore $Z_{\overline{1}}(B) \neq 0$ and $B$ is not simple as an algebra. Consequently $B=B_{\overline{0}}[u]=\mathbb{H}[u]=\mathbb{H} \oplus \mathbb{H} u, u^{2}=\alpha \neq 0$. Take $a \in \mathbb{H}$ and consider $W:=(V, a, u)$. It follows from (2) that $W$ is a subbimodule of $V$. Assume that $W=V$; then $V=((V, a, u), a, u)$. But we have for any $v \in V$ by super-linearized (4)

$$
((v, a, u), a, u)=((u, a, u), a, v)+[a, u](v, a, u)+[a, v](u, a, u)=0,
$$

a contradiction. Therefore $(V, \mathbb{H}, u)=0$, and by $(3)[u,(V, \mathbb{H}, \mathbb{H})] \subseteq(u, V, \mathbb{H})=0$. Let us show that if $[u, v]=0$, then $(u, u, v)=0$. In fact, by (10), $3(u, u, v)=$ $\left[u^{2}, v\right]-u[u, v]-[u, v] u=0$. Thus $((V, \mathbb{H}, \mathbb{H}), u, u)=0$.

Consider now the space $(V, u, u)$; it is also a subbimodule of $V$. Assume that $(V, u, u)=V$; then we have

$$
(V, \mathbb{H}, \mathbb{H})=((V, u, u), \mathbb{H}, \mathbb{H})=((V, \mathbb{H}, \mathbb{H}), u, u)=0 .
$$

We have already seen that $(V, \mathbb{H}, u)=0$ as well. Take $a, b \in \mathbb{H}$ and consider

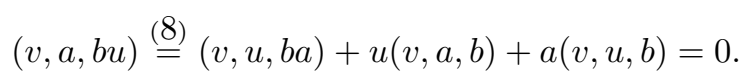

Therefore $(V, \mathbb{H}, B)=0$, which implies by (2) that $[V, \mathbb{H}] \subseteq V_{a s}=0$. Consequently, $V$ is an associative and commutative $\mathbb{H}$-bimodule. But it is impossible since for any $a, b \in \mathbb{H}$ and $v \in V$ we would have

$$
(a b) v=a(b v)=a(v b)=(v b) a=v(b a)=(b a) v,[a, b] v=v[a, b]=0,
$$

and $[a, b]=0$, a contradiction. Therefore, $(V, u, u)=0$.

It is easy now to see that $(V, u, B)=0$ and then $[V, u] \subseteq V_{a s}=0$. Therefore, $u \in Z(E(V, B))$ and $V=V_{\overline{0}}+V_{\overline{0}} u=V_{\overline{0}}[u]$. Clearly, $V_{\overline{0}}$ is an irreducible alternative H-bimodule. If $V_{\overline{0}}$ were associative, then $V$ would be associative as well, which is not the case. Therefore, $V \cong$ Cay $L$ where $L$ is a minimal left ideal of $\mathbb{H}$. As in the previous case, $A_{\overline{0}}$ is a primitive $P I$-algebra and $Z\left(A_{\overline{0}}\right)=Z(A)_{\overline{0}}$ is a field. Therefore $A=B$.

Assume now that $B=\mathbb{O}[u]=\mathbb{O} \oplus \mathbb{O} u, u^{2}=\lambda \in K, \lambda \neq 0$, where $\mathbb{O}$ is a central Cayley-Dickson algebra over $K$. As above, we have

$$
(V, u, \mathbb{O})=[u,(V, \mathbb{O}, \mathbb{O})]=((V, \mathbb{O}, \mathbb{O}), u, u)=0 .
$$

Assume that $(V, u, u)=V$; then $(V, \mathbb{O}, \mathbb{O})=0$ and $V$ is a unital associative bimodule over $\mathbb{O}$, which is impossible. Hence $(V, u, u)=0$. As above, we have $(V, u, \mathbb{O})=[V, u]=0$, hence $u \in Z_{\overline{1}}(E(V, B))$ and $V=V_{\overline{0}}[u]$. It is clear now that $V_{\overline{0}} \cong \operatorname{Reg} \mathbb{O}$. As in the proof of Theorem 4.1, we also have $A=B$.

Finally let $B=\mathbb{O}(4 \mid 4)$. Then $B_{\overline{0}}=\mathbb{H}$ and $B_{\overline{1}}$ is a Cayley bimodule over $\mathbb{H}$. By [19, Lemma 12], $V=V_{a} \oplus V_{c}$, where $V_{a}$ is an associative $\mathbb{H}$-bimodule and $V_{c}$ is a Cayley $\mathbb{H}$-bimodule. Moreover, by [9, Lemma 3.2], we have $V_{a} B_{\overline{1}}+B_{\overline{1}} V_{a} \subseteq$ $V_{c}, V_{c} B_{\overline{1}}+B_{\overline{1}} V_{c} \subseteq V_{a}$. Since $V$ is faithful, this implies that $V_{a} \neq 0, V_{c} \neq 0$. Let $Z_{a}:=\left\{v \in V_{a} \mid[v, \mathbb{H}]=0\right\}$. As in the proof of Lemma 3.3 in [9] we have that $Z_{a} \neq 0$ and $\left[Z_{a}, B\right]=0$. Furthermore, $3\left(Z_{a}, B, B\right) \subseteq\left[B B, Z_{a}\right]+B\left[B, Z_{a}\right]+\left[B, Z_{a}\right] B=0$. Hence $0 \neq Z_{a} \subseteq Z(E(V, B))$. Choose some homogeneous element $0 \neq u \in Z_{a}$; then the subspace $u \cdot B$ is a $B$-subbimodule of $V$ and the mapping $\varphi: a \mapsto u \cdot a$ is a $B$-bimodule homomorphism of $\operatorname{Reg} B$ onto $u B$, in the case when $u$ is even, or of $(\operatorname{Reg} B)^{\text {op }}$ onto $u B$, in the case when $u$ is odd. Since both $\operatorname{Reg} B$ and $(\operatorname{Reg} B)^{\text {op }}$ 
are irreducible, and $\varphi(1)=u \neq 0$, we have that $u B=V$ is isomorphic to $\operatorname{Reg} B$ or to $(\operatorname{Reg} B)^{\mathrm{op}}$. Clearly, in both cases we have $A=B$.

6.3. Superbimodules over the superalgebra $B(\Gamma, D, \gamma)$. In this section we consider the last remaining case of superbimodules in characteristic 3. Unlike the other cases, we cannot classify irreducible alternative superbimodules of characteristic 3 in any dimension, since the prime alternative superalgebras of characteristic 3 are classified only under certain restrictions.

Nevertheless, due to [10], every finite-dimensional non-associative prime alternative superalgebra $A$ is simple. Thus, if char $A=3$, then $A$ is one of the superalgebras $B(1 \mid 2), B(4 \mid 2), B(\Gamma, D, \gamma)$. The supermodules over the superalgebras $B(1 \mid 2), B(4 \mid 2)$ were classified in [9, 24] (see Theorems 2.3, 2.4). Therefore, to finish the classification of irreducible finite-dimensional superbimodules, it suffices to classify them over the superalgebra $B(\Gamma, D, \gamma)$. We will do this for any dimension.

Below $V$ denotes an irreducible faithful bimodule over the superalgebra $B=$ $B(\Gamma, D, \gamma)$ which is not associative.

We start by studying the structure of $V$ as a bimodule over $B_{\overline{0}}=\Gamma$.

Lemma 6.4. $(V, \Gamma, \Gamma)=[V, \Gamma]=0$.

Proof. Fix $a, b \in \Gamma$. Since $\Gamma$ is commutative and $(B, \Gamma, \Gamma)=0$, by (2) it follows that $(V, a, b)$ is a subbimodule of $V$. If $(V, a, b)=V$, then $V=(V, a, b)=((V, a, b), a, b)=$ 0 by (4). Thus, $(V, a, b)=0$.

Consider $[V, a]$. Since $[\Gamma, B]=0$ and $\operatorname{char} B=3$, it follows from (11) that $[V, a]$ is a subbimodule of $V$. Hence, $[V, a]=0$ or $[V, a]=V$.

Note that $D\left(a^{3}\right)=3 a^{2} D(a)=0$. We will show next that if $D(b)=0$ for some $b \in \Gamma$, then $[V, b]=0$.

Let $c, d \in \Gamma$. We have

$$
\begin{aligned}
(b, \bar{c}, \bar{d}) & =\overline{b c} \cdot \bar{d}-b \cdot(\gamma c d+2 D(c) d+c D(d)) \\
& =\gamma b c d+2 D(b c) d+b c D(d)-\gamma b c d-2 b D(c) d-b c D(d) \\
& =2 D(b c) d-2 b D(c) d=0 .
\end{aligned}
$$

It follows now from (2) that $(V, b, x)$ is a subbimodule of $V$ for any $x \in \Gamma$. If $(V, b, x)=V$, then $V=(V, b, x)=((V, b, x), b, x)=0$, by superized (6) $)$ Thus $(V, b, \bar{\Gamma})=0$.

If $[V, b]=V$, then again from (2) we get

$$
(V, B, B)=([V, b], B, B) \subseteq(B B, V, b)+B(B, V, b)+(B, V, b) B=0,
$$

which contradicts to the non-associativity of $V$. Hence, $[V, b]=0$.

Now, if $[V, a]=V$, then $V=[V, a]=[[V, a], a]=[[[V, a], a], a]=\left[V, a^{3}\right]=0$, by the above. Hence, $[V, a]=0$.

The next proposition is an adaptation of the results from [19] to the bimodule situation.

Proposition 6.1. Let $V=V_{\overline{0}} \oplus V_{\overline{1}}$ be an irreducible bimodule over an alternative superalgebra $A=A_{\overline{0}} \oplus A_{\overline{1}}$. If $N$ is a proper $A_{\overline{0}}$-subbimodule of $V_{i}$, and $\left(N, A_{\overline{1}}, A_{\overline{1}}\right) \subseteq$ $N$, then $V=\left(N \oplus A_{\overline{1}} N A_{\overline{1}}\right) \oplus\left(N A_{\overline{1}} \oplus A_{\overline{1}} N\right)$. Moreover, if $\left[N, A_{\overline{1}}\right]_{s}=0$, then $N=V_{i}$.

Proof. It is clear that $\left(N+A_{\overline{1}} N A_{\overline{1}}\right) \oplus\left(N A_{\overline{1}}+A_{\overline{1}} N\right)$ is a proper subbimodule of $V$ and hence it is equal to $V$. Let us prove that the sums in the summands are direct. Suppose that $N \cap A_{\overline{1}} N A_{\overline{1}}:=L \neq 0$. Then $L+L \circ A_{\overline{1}}$ is a non-zero subbimodule 
of $V$. Hence, $L+L \circ A_{\overline{1}}=V$, but this contradicts the fact that $L$ is a proper $A_{\overline{0}}$-subbimodule of $V_{i}$. Therefore, $N \cap A_{\overline{1}} N A_{\overline{1}}=0$.

Now let $L:=N A_{\overline{1}} \cap A_{\overline{1}} N$. Then $L A_{\overline{0}} \subseteq N A_{\overline{1}} \cdot A_{\overline{0}} \subseteq N A_{\overline{1}}$. On the other hand, $L A_{\overline{0}} \subseteq A_{\overline{1}} N \cdot A_{\overline{0}} \subseteq\left(A_{\overline{1}}, N, A_{\overline{0}}\right)+A_{\overline{1}} N \subseteq A_{\overline{1}} N$. Hence, $L A_{\overline{0}} \subseteq L$. Similarly, $A_{\overline{0}} L \subseteq L$. Thus $L+L \circ A_{\overline{1}}=V$. But $L A_{\overline{1}} \subseteq N A_{\overline{1}} \cdot A_{\overline{1}} \subseteq N$ and, similarly, $A_{\overline{1}} L \subseteq N$. Therefore $V=L+N$, a contradiction. So, $N A_{\overline{1}} \cap A_{\overline{1}} N=0$.

Finally, assume that $\left[N, A_{\overline{1}}\right]=0$. Then we have

$$
\left(A_{\overline{1}} N\right) A_{\overline{1}} \subseteq\left(N A_{\overline{1}}\right) A_{\overline{1}} \subseteq\left(N, A_{\overline{1}}, A_{\overline{1}}\right)+N A_{\overline{0}} \subseteq N,
$$

therefore $V=N \oplus N \circ A_{\overline{1}}$ and $N=V_{i}$.

Lemma 6.5. $\left(V_{\overline{0}}, \Gamma, \bar{\Gamma}\right)=0$ or $\left(V_{\overline{1}}, \Gamma, \bar{\Gamma}\right)=0$.

Proof. Let $W:=\{v \in V \mid(v, \Gamma, \bar{\Gamma})=0\}$. We show first that $W \neq 0$. Recall that $(\Gamma, \Gamma, \bar{\Gamma})=0$. By superization of (4), in view of Lemma 6.4 we have

$$
\begin{aligned}
((V, \Gamma, \bar{\Gamma}), \Gamma, \bar{\Gamma}) & \subseteq((\Gamma, \Gamma, \bar{\Gamma}), V, \bar{\Gamma})+((V, \bar{\Gamma}, \bar{\Gamma}), \Gamma, \Gamma) \\
& +((\Gamma, \bar{\Gamma}, \bar{\Gamma}), V, \Gamma)+[V, \bar{\Gamma}](\bar{\Gamma}, \Gamma, \Gamma) \subseteq(V, \Gamma, \Gamma)=0 .
\end{aligned}
$$

Therefore, $W \neq 0$. It follows from (2) that $W \Gamma \subseteq W$. Moreover, by (6) we have

$$
((W, \bar{\Gamma}, \bar{\Gamma}), \Gamma, \bar{\Gamma}) \subseteq(W, \Gamma, \bar{\Gamma})+(W, \bar{\Gamma}, \Gamma)=0 .
$$

Consequently, $(W, \bar{\Gamma}, \bar{\Gamma}) \subseteq W$. It is clear that $W$ is a graded subspace of $V$, and we see that the components $W_{i}, i=0,1$, satisfy the condition of Proposition 6.1. Consider $[W, \bar{\Gamma}]$. Since char $B=3$, it follows from (11) that $[W, \bar{\Gamma}]$ is a $\Gamma$-submodule of $V$. By superization of (7) and (8),

$$
\begin{aligned}
([W, \bar{\Gamma}], \bar{\Gamma}, \bar{\Gamma}) & \subseteq([\bar{\Gamma}, \bar{\Gamma}], \bar{\Gamma}, W)+[(\bar{\Gamma}, \bar{\Gamma}, \bar{\Gamma}), W]+[(\bar{\Gamma}, \bar{\Gamma}, W), \bar{\Gamma}] \\
\subseteq & (\Gamma, \bar{\Gamma}, W)+[W, \bar{\Gamma}]=[W, \bar{\Gamma}] .
\end{aligned}
$$

Consequently, the subspaces $\left[W_{i}, \bar{\Gamma}\right], i=0,1$, satisfy the condition of Proposition 6.1 as well.

Using (11) and Lemma 6.4 we obtain

$$
(\bar{\Gamma}[W, \bar{\Gamma}]) \bar{\Gamma} \subseteq([W, \bar{\Gamma}] \bar{\Gamma}) \bar{\Gamma} \subseteq[W, \bar{\Gamma}]+[W, \bar{\Gamma}] \Gamma \subseteq[W, \bar{\Gamma}] .
$$

Therefore, for every $i=0,1$, by Proposition 6.1 we have $\left[W_{i}, \bar{\Gamma}\right]=0$ or $V=$ $\left[W_{i}, \bar{\Gamma}\right] \bar{\Gamma}+\left[W_{i}, \bar{\Gamma}\right]$.

If $\left[W_{i}, \bar{\Gamma}\right]=0$, then by Proposition $6.1 W_{i}=V_{i}$ and $\left(V_{i}, \Gamma, \bar{\Gamma}\right)=0$.

In the second case we have $V_{1-i}=\left[W_{i}, \bar{\Gamma}\right]$. Then by (9), (10) we have

$$
\begin{aligned}
\left(V_{1-i}, \bar{\Gamma}, \Gamma\right) & =\left(\left[W_{i}, \bar{\Gamma}\right], \bar{\Gamma}, \Gamma\right) \subseteq[(\bar{\Gamma}, \bar{\Gamma}, \Gamma), W] \\
& +[(\bar{\Gamma}, \bar{\Gamma}, W), \Gamma]+([\Gamma, \bar{\Gamma}], \bar{\Gamma}, W) \subseteq[V, \Gamma]=0,
\end{aligned}
$$

hence $V_{1-i} \subseteq W$.

Lemma 6.6. If $\left(V_{\overline{0}}, \Gamma, \bar{\Gamma}\right)=0$, then $\left[V_{\overline{0}}, \bar{\Gamma}\right]=0$.

Proof. Similarly as it was done for $W$ in the proof of Lemma 6.5, we can show that $\left[V_{\overline{0}}, \bar{\Gamma}\right]=0$ or $V=\left[V_{\overline{0}}, \bar{\Gamma}\right] \bar{\Gamma}+\left[V_{\overline{0}}, \bar{\Gamma}\right]$. Consider the last case. As in the proof of Lemma 6.5 we obtain that $\left(V_{\overline{1}}, \Gamma, \bar{\Gamma}\right)=\left(\left[V_{\overline{0}}, \bar{\Gamma}\right], \Gamma, \bar{\Gamma}\right)=0$, so $(V, \Gamma, \bar{\Gamma})=0$.

Note that for any non-zero $a \in \Gamma$ we have $V a=V$, hence $V=V a=(V a) a=$ $V a^{3}$. Thus $a^{3} \neq 0$. Since $\Gamma$ is $D$-simple [19, it follows from S. Yuan 21] that $\Gamma$ is a field. 
By (2) we have

$$
0=\left(V_{\overline{0}}, \Gamma, \bar{\Gamma}\right)=\left(\left[V_{\overline{0}}, \bar{\Gamma}\right] \bar{\Gamma}, \Gamma, \bar{\Gamma}\right)=\left[V_{\overline{0}}, \bar{\Gamma}\right](\bar{\Gamma}, \Gamma, \bar{\Gamma}) .
$$

In particular, for any $a \in \Gamma$ we have $0=\left[V_{0}, \bar{\Gamma}\right](a, \overline{1}, \overline{1})=2\left[V_{0}, \bar{\Gamma}\right] D(a)$. (It was proved by E. C. Posner 13 that any $D$-simple algebra contains an identity element 1.) By choosing $a$ such that $D(a) \neq 0$ we obtain that $\left[V_{0}, \bar{\Gamma}\right]=0$.

Let $(A, D)$ be an algebra with a derivation $D$. An $A$-bimodule $(V, d)$ with a linear mapping $d: V \rightarrow V$ is called a bimodule with derivation or a D-bimodule over the algebra $(A, D)$, if the linear mapping $D+d: a+v \mapsto D(a)+d(v)$ is a derivation of the split null extension $E=A \oplus V$. If, in addition, $V$ has no proper $d$-invariant $A$-subbimodules, then $(V, d)$ is called a $D$-simple $A$-bimodule.

Let $(V, d)$ be an associative and commutative $D$-bimodule over an algebra with a derivation $(\Gamma, D)$. Consider the split null extension $E=\Gamma \oplus V$ with the derivation $D+d$ and construct the alternative superalgebra $B(E, D+d, \gamma)$. We have

$$
B(E, D+d, \gamma)=(\Gamma \oplus V) \oplus \overline{\Gamma \oplus V}=(\Gamma \oplus \bar{\Gamma}) \oplus(V \oplus \bar{V})=B \oplus W,
$$

where $W:=V \oplus \bar{V}$ is an alternative bimodule over the superalgebra $B=B(\Gamma, D, \gamma)$. We denote this bimodule by $B(V, d, \gamma)$.

From the definition, it is easy to recover the explicit action of $B(\Gamma, D, \gamma)$ on $B(V, d, \gamma)$ :

$$
\begin{array}{r}
a \cdot v=v \cdot a=a v, \quad \bar{a} \cdot v=v \cdot \bar{a}=a \cdot \bar{v}=\bar{v} \cdot a=\overline{a v}, \\
\bar{a} \cdot \bar{v}=2 D(a) v+a d(v)+\gamma a v, \quad \bar{v} \cdot \bar{a}=2 d(v) a+v D(a)+\gamma v a .
\end{array}
$$

We are now ready to prove

Theorem 6.2. Let $V$ be an irreducible faithful non-associative alternative bimodule over the simple superalgebra $B(\Gamma, D, \gamma)$. Then, up to the changing of parity, $\left(V_{\overline{0}}, d\right)$ is a D-simple faithful associative and commutative bimodule over the algebra with derivation $(\Gamma, D)$, where $d(v)=-(v, \overline{1}, \overline{1})$ is a derivation of $V_{\overline{0}} ; V_{\overline{1}}=V_{\overline{0}} \cdot \overline{1}$ is an isomorphic copy of $V_{\overline{0}}$ and $V \cong B\left(V_{\overline{0}}, d, \gamma\right)$.

Proof. By Lemmas 6.4, 6.5, 6.6, we may assume, up to the changing of the parity, that $\left(V_{\overline{0}}, \Gamma, B\right)=\left[V_{\overline{0}}, B\right]=0$. Let $d: V_{\overline{0}} \rightarrow V_{\overline{0}}, d: v \mapsto 2(v, \overline{1}, \overline{1})$; then it follows from (2) that $\left(V_{\overline{0}}, d\right)$ is an associative and commutative $D$-bimodule over $(\Gamma, D)$. For $v \in V_{\overline{0}}$, denote $\bar{v}=v \cdot \overline{1}$; then we have

$$
\begin{aligned}
a \bar{v} & =a(\overline{1} v)=(a \overline{1}) v=\bar{a} v=(\overline{1} a) v=\overline{1}(a v)=\overline{a v}, \\
\bar{a} \bar{v} & =\bar{a}(\overline{1} v)=(\bar{a} \overline{1}) v-(\bar{a}, \overline{1}, v)=(2 D(a)+\gamma a) v-(a \overline{1}, \overline{1}, v) \\
& \underline{\equiv}=(2 D(a)+\gamma a) v-(\overline{1} \cdot \overline{1}, a, v)-(\overline{1}, \overline{1}, v) a-(\overline{1}, a, v) \overline{1} \\
& =2 D(a) v+d(v) a+\gamma a v,
\end{aligned}
$$

and similarly $\bar{v} \bar{a}=2 d(v) a+v D(a)+\gamma v a$. Therefore, $V \cong B\left(V_{\overline{0}}, d, \gamma\right)$.

The converse is also true:

Proposition 6.2. For any D-simple faithful associative and commutative bimodule $(V, d)$ over $(\Gamma, D)$ and any $\gamma \in \Gamma$ the bimodule $B(V, d, \gamma)$ is an irreducible faithful alternative superbimodule over the simple superalgebra $B=B(\Gamma, D, \gamma)$. Moreover, the $B$-superbimodules $B(V, d, \gamma)$ and $B\left(V^{\prime}, d^{\prime}, \gamma\right)$ are isomorphic if and only if the differential bimodules $(V, d)$ and $\left(V^{\prime}, d^{\prime}\right)$ over $(\Gamma, D)$ are isomorphic. 
Proof. Let $W$ be a non-zero subbimodule of $V$; then $W=W_{\overline{0}} \oplus \bar{U}$ for some $\Gamma$ subbimodule $U$ of $V_{\overline{0}}$. We show that $W_{\overline{0}} \neq 0$. Assume to the contrary that $W_{\overline{0}}=0$. Then $\bar{v} \cdot \overline{1}=\overline{1} \cdot \bar{v}=0$ for any $\bar{v} \in W_{\overline{1}}=\bar{U}$. Hence $d(v)=0$ for any $v \in U$. It follows that $U$ is a non-zero $D$-submodule of $V_{\overline{0}}$. Thus $U=V_{\overline{0}}$ and $0=d(v a)=v D(a)$ for any $v \in V_{\overline{0}}, a \in \Gamma$. Consequently, $D(\Gamma)=0$, a contradiction.

Therefore $W_{\overline{0}}$ is a non-zero $\Gamma$-subbimodule of $V_{\overline{0}}$ and $\overline{W_{\overline{0}}} \subseteq W_{\overline{1}}$. For any $v \in W_{\overline{0}}$, $\overline{1} \cdot \bar{v}=\gamma v+d(v) \in W_{\overline{0}}$. Hence $d\left(W_{\overline{0}}\right) \subseteq W_{\overline{0}}$ and $W_{\overline{0}}=V_{\overline{0}}$.

Finally, if the superbimodules $B(V, d, \gamma)$ and $B\left(V^{\prime}, d^{\prime}, \gamma\right)$ are isomorphic, then their even parts $V_{\overline{0}}$ and $V_{\overline{0}}^{\prime}$ are isomorphic associative and commutative bimodules over $\Gamma$. Moreover, the derivations $d$ and $d^{\prime}$ are defined via the same mapping $v \mapsto 2(v, \overline{1}, \overline{1})$, hence $(V, d) \cong\left(V^{\prime}, d^{\prime}\right)$.

Clearly, for any $D$-simple algebra $(\Gamma, D)$ the regular bimodule with derivation $\operatorname{Reg}(\Gamma, D)$ is a $D$-simple bimodule over $(\Gamma, D)$. Moreover, for any $a \in \Gamma$ the map $D+R_{a}: \Gamma \rightarrow \Gamma$ is a derivation of the bimodule $\operatorname{Reg} \Gamma$. In fact, for any $v \in \operatorname{Reg} \Gamma, b \in \Gamma$ we have

$\left(D+R_{a}\right)(v b)=D(v b)+v b a=D(v) b+v D(b)+v b a=\left(D+R_{a}\right)(v) b+v D(b)$.

It is clear that the differential bimodule $\left(\operatorname{Reg} \Gamma, D+R_{a}\right)$ is $D$-simple as well.

Proposition 6.3. The D-bimodules $\left(\operatorname{Reg} \Gamma, D+R_{a}\right)$ and $\left(\operatorname{Reg} \Gamma, D+R_{b}\right)$ over $(\Gamma, D)$ are isomorphic if and only if there exists an invertible $c \in \Gamma$ such that $D(c)=(a-b) c$. In particular, if $a, b \in F$, then $\left(\operatorname{Reg} \Gamma, D+R_{a}\right) \cong\left(\operatorname{Reg} \Gamma, D+R_{b}\right)$ if and only if $a-b \in \operatorname{Spec} D$.

Proof. Let $\varphi:\left(\operatorname{Reg} \Gamma, D+R_{a}\right) \rightarrow\left(\operatorname{Reg} \Gamma, D+R_{b}\right)$ be an isomorphism of $D$ bimodules over $(\Gamma, D)$. Let $c:=\varphi(1)$; then for any $x \in \Gamma$ we have $\varphi(x)=\varphi(1 \cdot x)=$ $\varphi(1) x=c x$. Since $\varphi$ is an isomorphism, $c \Gamma=\Gamma$ and $c$ is invertible. Furthermore,

$$
\begin{aligned}
\varphi\left(\left(D+R_{a}\right)(x)\right) & =c D(x)+c a x=\left(D+R_{b}\right)(\varphi(x))=D(c x)+b c x \\
& =D(c) x+c D(x)+b c x,
\end{aligned}
$$

which gives $(D(c)-(a-b) c) \Gamma=0$. Since $\Gamma$ is $D$-simple, this proves that $D(c)=$ $(a-b) c$. Conversely, if $c$ is an invertible element that satisfies this relation, then the map $x \mapsto c x$ is an isomorphism of $D$-bimodules $\left(\operatorname{Reg} \Gamma, D+R_{a}\right)$ and $\left(\operatorname{Reg} \Gamma, D+R_{b}\right)$. It remains to notice that if $\lambda \in \operatorname{Spec} D$, that is, $D(c)=\lambda c$ for some $c \in \Gamma$, then $c \Gamma$ is a $D$-ideal in $\Gamma$, hence $c \Gamma=\Gamma$ and $c$ is invertible.

We now describe the faithful $D$-simple bimodules over a finite-dimensional $D$ simple associative commutative algebra $(\Gamma, D)$ in the case when the ground field $F$ is algebraically closed. Observe that due to S. Yuan [21] in this case $\Gamma \cong$ $F\left[t_{1}, \ldots, t_{n}\right] /\left(t_{1}^{3}, \ldots, t_{n}^{3}\right)$.

Theorem 6.3. Let $(\Gamma, D)$ be a D-simple associative commutative algebra over an algebraically closed field $F$ of characteristic 3 , and let $(V, d)$ be a $D$-simple finitedimensional associative and commutative bimodule over $(\Gamma, D)$. Then there exist $\lambda \in F$ and $v \in V$ such that $V=v \Gamma$ and the action of the derivation $d$ is given by $d(v a)=v(\lambda a+D(a))$. In other words, the bimodule $(v \Gamma, d)$ is isomorphic to the $D$-bimodule $(\operatorname{Reg} \Gamma, D+\lambda)$. The parameter $\lambda$ is defined uniquely modulo Spec D.

Proof. Since the field $F$ is algebraically closed and $V$ is finite-dimensional, the linear operator $d: V \rightarrow V$ has an eigenvector $v$ with an eigenvalue $\lambda \in F$. For any 
$a \in \Gamma$ we have $d(v a)=d(v) a+v D(a)=v(\lambda a+D(a))$, which proves that $v \Gamma$ is a $d$-subbimodule of $V$. Clearly, $v \Gamma \neq 0$ and therefore $V=v \Gamma$.

Consider the mapping $\varphi: \operatorname{Reg} \Gamma \rightarrow V, a \mapsto v a$. Clearly, $\varphi$ is an isomorphism of $\Gamma$-modules. Moreover, we have

$$
\varphi((D+\lambda)(a))=v(D(a)+\lambda a)=d(v a)=d(\varphi(a))
$$

which shows that $(V, d) \cong(\operatorname{Reg} \Gamma, D+\lambda)$. The last statement follows from Proposition 6.3 .

As a result, for the finite-dimensional alternative superalgebras over an algebraically closed field we have a complete description of irreducible non-trivial bimodules, which are not associative.

Theorem 6.4. Let $A$ be a finite-dimensional alternative superalgebra over an algebraically closed field $F$ and let $V$ be an irreducible faithful non-trivial alternative superbimodule over $A$, which is not associative. Then either $\operatorname{dim} A \leq 2, A_{\overline{1}}=$ $F x, x^{2}=0$ and $V \cong V^{\varepsilon}(1 \mid 1), \varepsilon^{2}+\varepsilon+1=0$, or the superalgebra $A$ is simple and $V$ is either isomorphic to one of the bimodules $\operatorname{Reg} A,(\operatorname{Reg} A)^{\mathrm{op}}$, or one of the following cases holds:

- $A=F[\sqrt{1}]$ and $V$ is one of the 2-dimensional bimodules of types (1) - (3) described in Theorem 2.2,

- $\operatorname{char} F=3, A=B(1 \mid 2), V=V_{\lambda, \mu}(3 \mid 3)$;

- $\operatorname{char} F=3, A=B(\Gamma, D, \gamma), V=B(\operatorname{Reg} \Gamma, D+\lambda, \gamma), \lambda \notin \operatorname{Spec} D$;

- char $F=2, A=\mathrm{M}_{1 \mid 1}(F), V=$ Cay $L, L$ is a minimal $\mathbb{Z}_{2}$-graded left ideal of $\mathrm{M}_{1 \mid 1}(F)$;

- $\operatorname{char} F=2, A=\mathrm{M}_{2}(F)[\sqrt{1}], V=($ Cay $L)[\sqrt{1}], L$ is a minimal left ideal of $\mathrm{M}_{2}(F)$.

Observe that there is a misprint in the announcement of this result in [25]: the case $A=B(\Gamma, D, \gamma)$ is omitted.

We conclude with some comments on the remaining open case of classification of infinite-dimensional irreducible superbimodules $V$ over non-simple prime superalgebras $A$ of characteristic 3 .

If $A$ is "of classical type", that is, $A$ is a central order in a simple superalgebra $B$, then due to Lemma $6.2 \mathrm{~V}$ is an irreducible superbimodule over $B$ and the structure of $V$ is known. But when $A$ is exceptional, we could not determine the structure of $V$ and do not know whether such bimodules exist. One of the problems which appears in the proof is related to the structure of differentially simple modules over associative algebras with derivations. In particular, if a hypothetic irreducible superbimodule $V$ exists, then there exists an associative locally nilpotent algebra $C$ with a set of derivations $\left\{D_{\alpha}\right\}$ which has an irreducible differential module. Answering the question of one of the authors, Agata Smoktunowicz and Michal Ziembowski recently showed in 23 ] that in the case of one derivation such modules exist.

\section{ACKNOWLEDGEMENT}

We thank the referee for various useful suggestions. 


\section{REFERENCES}

[1] Yuri Bahturin, Antonio Giambruno, and David M. Riley, Group-graded algebras with polynomial identity, Israel J. Math. 104 (1998), 145-155, DOI 10.1007/BF02897062. MR1622291 (99c:16041)

[2] George M. Bergman, The diamond lemma for ring theory, Adv. in Math. 29 (1978), no. 2, 178-218, DOI 10.1016/0001-8708(78)90010-5. MR.506890 (81b:16001)

[3] L. A. Bokut', Imbeddings into simple associative algebras (Russian), Algebra i Logika 15 (1976), no. 2, 117-142, 245. MR0506423 (58 \#22167)

[4] L. A. Bokut' and P. S. Kolesnikov, Gröbner-Shirshov bases: from inception to the present time (Russian, with English and Russian summaries), Zap. Nauchn. Sem. S.-Peterburg. Otdel. Mat. Inst. Steklov. (POMI) 272 (2000), no. Vopr. Teor. Predst. Algebr i Grupp. 7, 26-67, 345, DOI 10.1023/A:1023490323855; English transl., J. Math. Sci. (N. Y.) 116 (2003), no. 1, 2894-2916. MR.1811792 (2002a:16030)

[5] R. H. Bruck and Erwin Kleinfeld, The structure of alternative division rings, Proc. Amer. Math. Soc. 2 (1951), 878-890. MR0045099 (13,526e)

[6] M. Cohen and S. Montgomery, Group-graded rings, smash products, and group actions, Trans. Amer. Math. Soc. 282 (1984), no. 1, 237-258, DOI 10.2307/1999586. MR728711(85i:16002)

[7] Alberto Elduque and Ivan P. Shestakov, Irreducible non-Lie modules for Malcev superalgebras, J. Algebra 173 (1995), no. 3, 622-637, DOI 10.1006/jabr.1995.1106. MR.1327872 (96e:17070)

[8] N. Jacobson, Structure of alternative and Jordan bimodules, Osaka Math. J. 6 (1954), 1-71. MR.0064762 (16,330d)

[9] M. C. López-Díaz and Ivan P. Shestakov, Representations of exceptional simple alternative superalgebras of characteristic 3, Trans. Amer. Math. Soc. 354 (2002), no. 7, 2745-2758 (electronic), DOI 10.1090/S0002-9947-02-02993-8. MR.1895201(2003b:17039)

[10] M. C. López-Díaz and Ivan P. Shestakov, Alternative superalgebras with DCC on twosided ideals, Comm. Algebra 33 (2005), no. 10, 3479-3487, DOI 10.1080/AGB-200058391. MR2175446 (2006g:17034)

[11] N. A. Pisarenko, The structure of alternative superbimodules (Russian, with Russian summary), Algebra i Logika 33 (1994), no. 6, 689-707, 717, DOI 10.1007/BF00756352; English transl., Algebra and Logic 33 (1994), no. 6, 386-397 (1995). MR1347266 (96h:17042)

[12] S. V. Polikarpov and I. P. Shestakov, Nonassociative affine algebras, Algebra and Logic 29 (1990), no. 6, 458-466 (1991), DOI 10.1007/BF01978558. MR.1159142 (93b:17009)

[13] Edward C. Posner, Differentiably simple rings, Proc. Amer. Math. Soc. 11 (1960), 337-343. MR0113908 (22 \#4739)

[14] Louis Halle Rowen, Polynomial identities in ring theory, Pure and Applied Mathematics, vol. 84, Academic Press, Inc. [Harcourt Brace Jovanovich, Publishers], New York-London, 1980. MR.576061 (82a:16021)

[15] R. D. Schafer, Alternative algebras over an arbitrary field, Bull. Amer. Math. Soc. 49 (1943), 549-555. MR0008610 (5,33d)

[16] R. D. Schafer, Representations of alternative algebras, Trans. Amer. Math. Soc. 72 (1952), 1-17. MR0045101 (13,527b)

[17] I. P. Shestakov, Absolute zero divisors and radicals of finitely generated alternative algebras (Russian), Algebra i Logika 15 (1976), no. 5, 585-602, 606. MR0506525 (58 \#22218)

[18] I. P. Shestakov, Superalgebras and counterexamples (Russian), Sibirsk. Mat. Zh. 32 (1991), no. 6, 187-196, 207, DOI 10.1007/BF00971214; English transl., Siberian Math. J. 32 (1991), no. 6, 1052-1060 (1992). MR1156760 (93c:17006)

[19] I. P. Shestakov, Prime alternative superalgebras of arbitrary characteristic (Russian, with Russian summary), Algebra i Logika 36 (1997), no. 6, 675-716, 722, DOI 10.1007/BF02671556; English transl., Algebra and Logic 36 (1997), no. 6, 389-412. MR.1657313 (99k:17006)

[20] I. P. Shestakov, Alternative and Jordan superalgebras (Russian, with Russian summary), Algebra, geometry, analysis and mathematical physics (Russian) (Novosibirsk, 1996), Izdat. Ross. Akad. Nauk Sib. Otd. Inst. Mat., Novosibirsk, 1997, pp. 157-169, 191. MR.1624131 (99g:17009)

[21] Shuen Yuan, Differentiably simple rings of prime characteristic, Duke Math. J. 31 (1964), 623-630. MR0167499 (29 \#4772) 
[22] A. M. Slin'ko and I. P. Shestakov, Right representations of algebras (Russian), Algebra i Logika 13 (1974), no. 5, 544-588, 605-606. MR0498761 (58 \#16810)

[23] Agata Smoktunowicz and Michał Ziembowski, Differential polynomial rings over locally nilpotent rings need not be Jacobson radical, J. Algebra 412 (2014), 207-217, DOI 10.1016/j.jalgebra.2014.04.022. MR.3215954

[24] M. N. Trushina, Irreducible alternative superbimodules over the simple alternative superalgebra $B(1,2)$ (Russian, with English and Russian summaries), Fundam. Prikl. Mat. 7 (2001), no. 3, 897-908. MR1879305(2003g:17045)

[25] M. N. Trushina and I. P. Shestakov, Representations of alternative algebras and superalgebras (Russian, with English and Russian summaries), Fundam. Prikl. Mat. 17 (2011/12), no. 1, 233-246, DOI 10.1007/s10958-012-0932-y; English transl., J. Math. Sci. (N. Y.) 185 (2012), no. 3, 504-512. MR2898228

[26] C. T. C. Wall, Graded Brauer groups, J. Reine Angew. Math. 213 (1963/1964), 187-199. MR0167498 (29 \#4771)

[27] Oscar Zariski and Pierre Samuel, Commutative algebra, Volume I, The University Series in Higher Mathematics, D. Van Nostrand Company, Inc., Princeton, New Jersey, 1958. With the cooperation of I. S. Cohen. MR0090581 (19,833e)

[28] E. I. Zel'manov and I. P. Shestakov, Prime alternative superalgebras and the nilpotency of the radical of a free alternative algebra (Russian), Izv. Akad. Nauk SSSR Ser. Mat. 54 (1990), no. 4, 676-693; English transl., Math. USSR-Izv. 37 (1991), no. 1, 19-36. MR.1073082 $(91 \mathrm{j}: 17003)$

[29] K. A. Zevlakov, The radical and representations of alternative rings (Russian), Algebra i Logika 11 (1972), 162-173, 237. MR0325723 (48 \#4070)

[30] K. A. Ževlakov, A. M. Slin'ko, I. P. Shestakov, and A. I. Širšov, Koltsa, blizkie k assotsiativnym (Russian), "Nauka", Moscow, 1978. Sovremennaya Algebra. [Monographs in Modern Algebra]. MR518614 (80h:17002)

[31] Max Zorn, Theorie der alternativen ringe (German), Abh. Math. Sem. Univ. Hamburg 8 (1931), no. 1, 123-147, DOI 10.1007/BF02940993. MR3069547

[32] Max Zorn, Alternative rings and related questions I: existence of the radical, Ann. of Math. (2) 42 (1941), 676-686. MR0005098 (3,100e)

Institute of Mathematics and Statistics, University of São Paulo, Rua do Matão, 1010-CEP 05508-090, SÃo PAUlo, Brazil

Institute of Mathematics, Informatics and Natural Sciences, Moscow City Pedagogical University, Ul. Sheremetevskaya, 29, Moscow, 129226, Russia 